\title{
Water Wells on Isla de Vieques, \\ Puerto Rico
}

By GREGORY S. CHERRY and JUAN RAMOS

U.S. Geological Survey

Open-File Report 95-368

Prepared in cooperation with the

U.S. OFFICE OF MANAGEMENT AND BUDGET

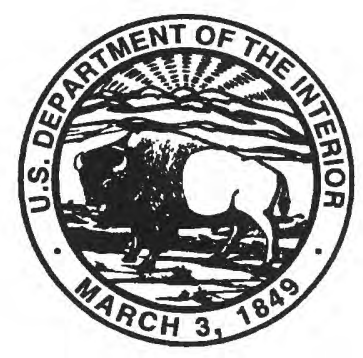

San Juan, Puerto Rico

1995 


\title{
U.S. DEPARTMENT OF THE INTERIOR \\ BRUCE BABBITT, Secretary
}

\author{
U.S. GEOLOGICAL SURVEY \\ Gordon P. Eaton, Director
}

For additional information write to:

Copies of this report can be purchased from:

Chief, Caribbean District

U.S. Geological Survey

Water Resources Division

GSA Center

651 Federal Drive, Suite 400-15

Guaynabo, Puerto Rico 00965
U.S. Geological Survey

Earth Science Information Center

Open-File Reports Section

Box 25286, MS 517

Denver Federal Center

Denver, CO 80225 


\section{CONTENTS}

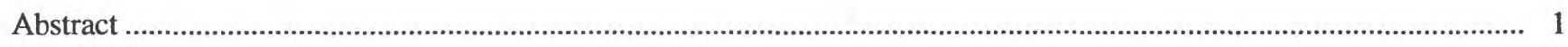

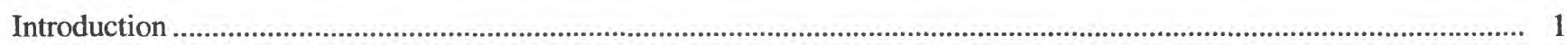

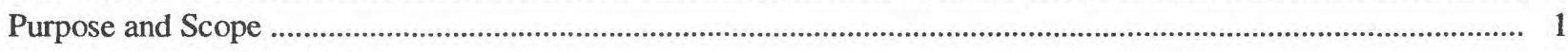

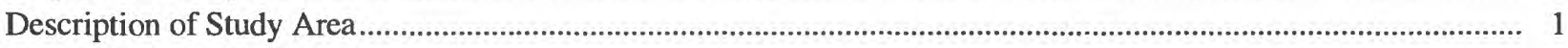

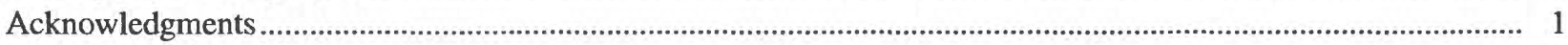

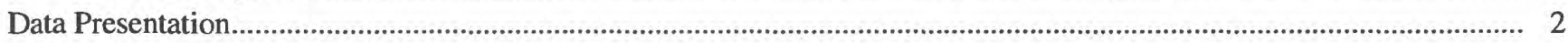

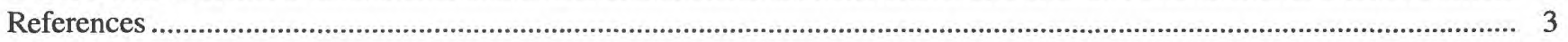

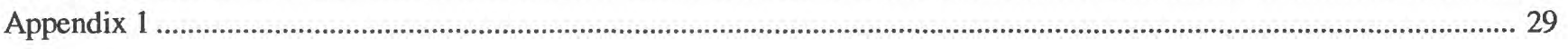

FIGURES

1. Map showing location of Isla de Vieques, Puerto Rico, and area grids............................................................... 2

2-14. Maps showing location of wells in area grids, Isla de Vieques, Puerto Rico:

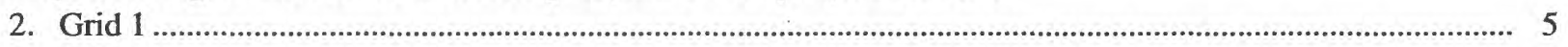

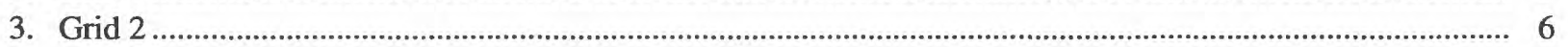

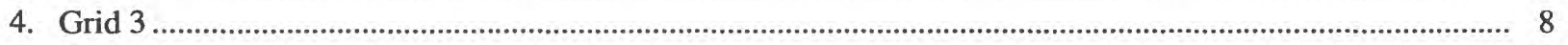

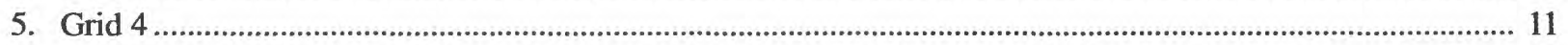

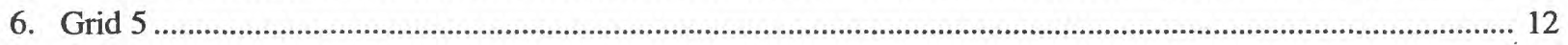

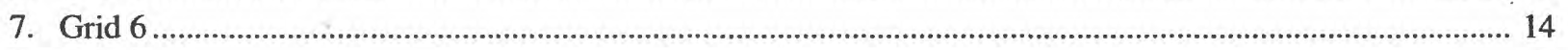

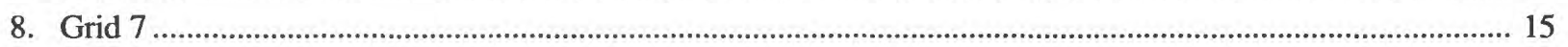

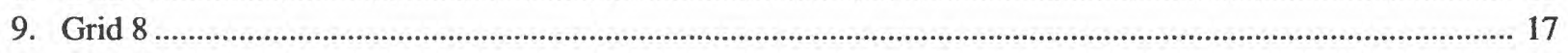

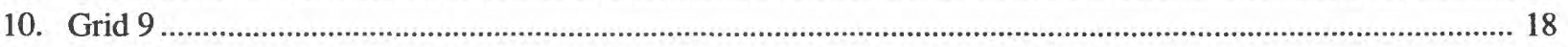

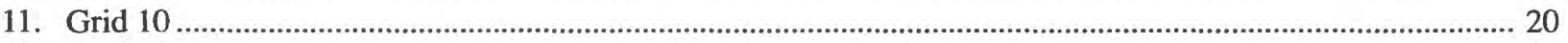

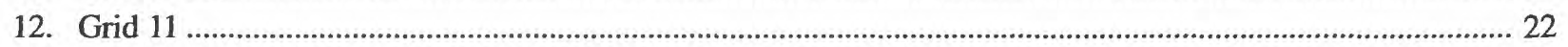

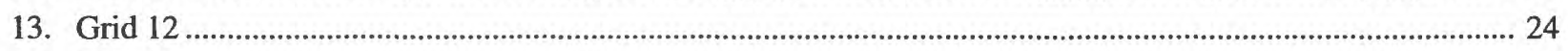

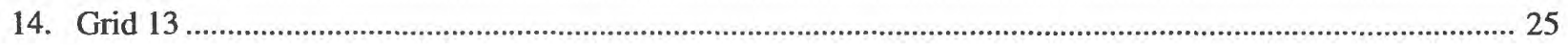

\section{TABLES}

1-6. Description of wells in area grids, Isla de Vieques, Puerto Rico:

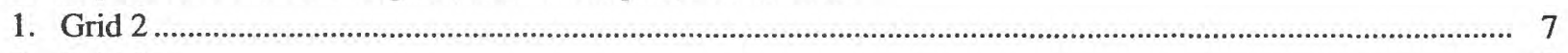

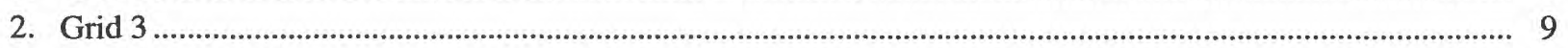

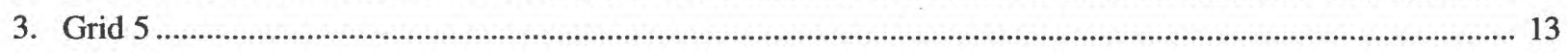

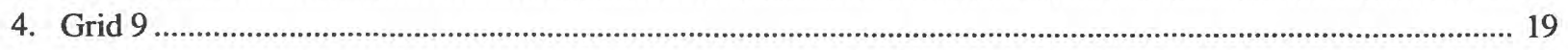

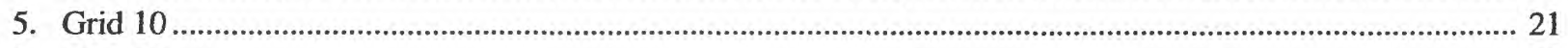

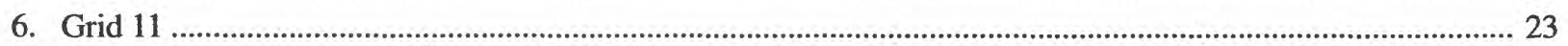


CONVERSION FACTORS, ABBREVIATED WATER-QUALITY UNITS, AND ACRONYMS

\begin{tabular}{rll}
\hline Multiply & \multicolumn{1}{c}{ By } & To obtain \\
\hline foot & 0.3048 & meter \\
gallon & 3.785 & liter \\
gallon per day & 0.003785 & cubic meter per day \\
gallon per minute & 0.06308 & liter per second \\
inch & 25.4 & millimeter \\
mile & 1.609 & kilometer \\
square mile & 259.0 & hectare \\
Temperature is given in degree Celsius $\left({ }^{\circ} \mathrm{C}\right)$, which can be converted to degree Fahrenheit $\left({ }^{\circ} \mathrm{F}\right)$ \\
\end{tabular}

\section{Abbreviated water-quality units used in this report:}

microsiemen per centimeter at 25 degrees Celsius $(\mu \mathrm{S} / \mathrm{cm})$

milligram per liter $(\mathrm{mg} / \mathrm{L})$

Acronyms used in this report:

PRASA Puerto Rico Aqueduct and Sewer Authority

PRIDCO Puerto Rico Industrial Development Corporation

USGS U.S. Geological Survey

USOMB U.S. Office of Management and Budget 


\title{
Water Wells on Isla de Vieques, Puerto Rico
}

\author{
By Gregory S. Cherry and Juan Ramos
}

\begin{abstract}
This report presents a compilation of wellinventory data collected from August through October 1991 on Isla de Vieques, Puerto Rico. The report includes maps depicting well locations and tables of well-inventory data for 73 wells. Currently (1995), the demand for freshwater on Isla de Vieques is met by a pipeline from eastern Puerto Rico and by rooftop-rainfall catchment basins or cisterns. The pipeline, constructed in 1977, replaced the Esperanza Valley well field as a source of public supply. The Esperanza Valley well field was shut down in 1978 due to increasing salinity and maintenance problems. The wellinventory data in this report provides a data base to assist hydrologists, water managers, and planners in the utilization of the limited ground-water resources of Isla de Vieques.
\end{abstract}

\section{INTRODUCTION}

The principal aquifer on Isla de Vieques is in the alluvial deposits of the Esperanza Valley in the southcentral part of the island (fig. 1). The Puerto Rico Aqueduct and Sewer Authority (PRASA) is responsible for freshwater supply and distribution to the public for Isla de Vieques. To meet the demand for freshwater on Isla de Vieques, a pipeline from eastern Puerto Rico provides 800,000 gallons per day to the residents of the island (W. Conde, Puerto Rico Aqueduct and Sewer Authority, personal commun., 1992). Prior to the construction of the freshwater pipeline in 1977, the primary source of freshwater was the Esperanza Valley well field ( 13 wells) operated by the PRASA. These wells produced a combined yield of 400,000 gallons per day for public supply until overpumpage resulted in the intrusion of saline water into the wells near the coast (Torres-González, 1989). If properly maintained and utilized, the well field in the Esperanza Valley could augment water from the pipeline and provide the island with an alternative source of freshwater in the event of emergencies.

\section{Purpose and Scope}

In 1990, the U.S. Geological Survey (USGS) began a cooperative investigation with the U.S. Office of Management and Budget (USOMB) to study and develop the ground-water resources on Isla de Vieques, Puerto Rico. This report presents data from 73 wells surveyed in the field from August through October 1991, including the specific conductance and chloride concentration in water from selected wells.

\section{Description of Study Area}

Isla de Vieques is the largest offshore island (about 51 square miles) belonging to the Commonwealth of Puerto Rico and is located about 7 miles east of Puerto Rico and 9 miles south of Isla de Culebra (fig. 1). The topography of Isla de Vieques is characterized by low rounded hills and small valleys with the higher elevations at the center of an east-west trending ridge (Meyerhoff, 1927). The highest peak is Monte Pirata, in the southwest, with an elevation of 987 feet.

\section{Acknowledgments}

The authors express their gratitude to the many well and landowners on Isla de Vieques for their assistance in locating wells and allowing access to their property. A special thanks goes to Wilfredo Conde of the PRASA for his support and assistance during the well rehabilitation phase of the study. 
DATA PRESENTATION

The locations, descriptions, and water-quality data (specific conductance and chloride concentration) of 73 wells constructed on Isla de Vieques are presented in this report. For ease in locating wells, Isla de Vieques was divided into 13 grids (fig. 1). All grids shown in figure 1 are presented as separate figures in this report (figs. 2-14). Grid 7 (fig. 8) represents a 1.5by 2.5 -minute section; the remaining grids represent 2.5-minute square sections of the USGS topographic map of Isla de Vieques. Each figure is published at the original topographic map scale of 1:30,000. Some grids cover areas in which no wells exist.

Tables 1 to 6 contain well information for grids that have wells located in them. Information presented in these tables includes the well number as referenced on the preceding figure, well name, use of water, year of construction, measured depth of well, well casing diameter, type of well finish and finish interval, landsurface altitude, date water level was measured, depth to water below land-surface datum, and specific conductance and chloride concentration determined from the water sample. The well numbers used in tables 1 through 6 consist of two numbers, the first referring to the grid number in figure 1 and the second to the number designation in the grid. Wells located in close proximity to one another will have the same well number followed by a letter designation. The distance between the wells is noted in the remarks section of the appendix. Wells listed in this report can be crossreferenced in other USGS publications by the siteidentification number that appears in the appendix. The site-identification number conforms with the USGS Ground-Water Site Inventory (GWSI) classification system; once this number is established for a given

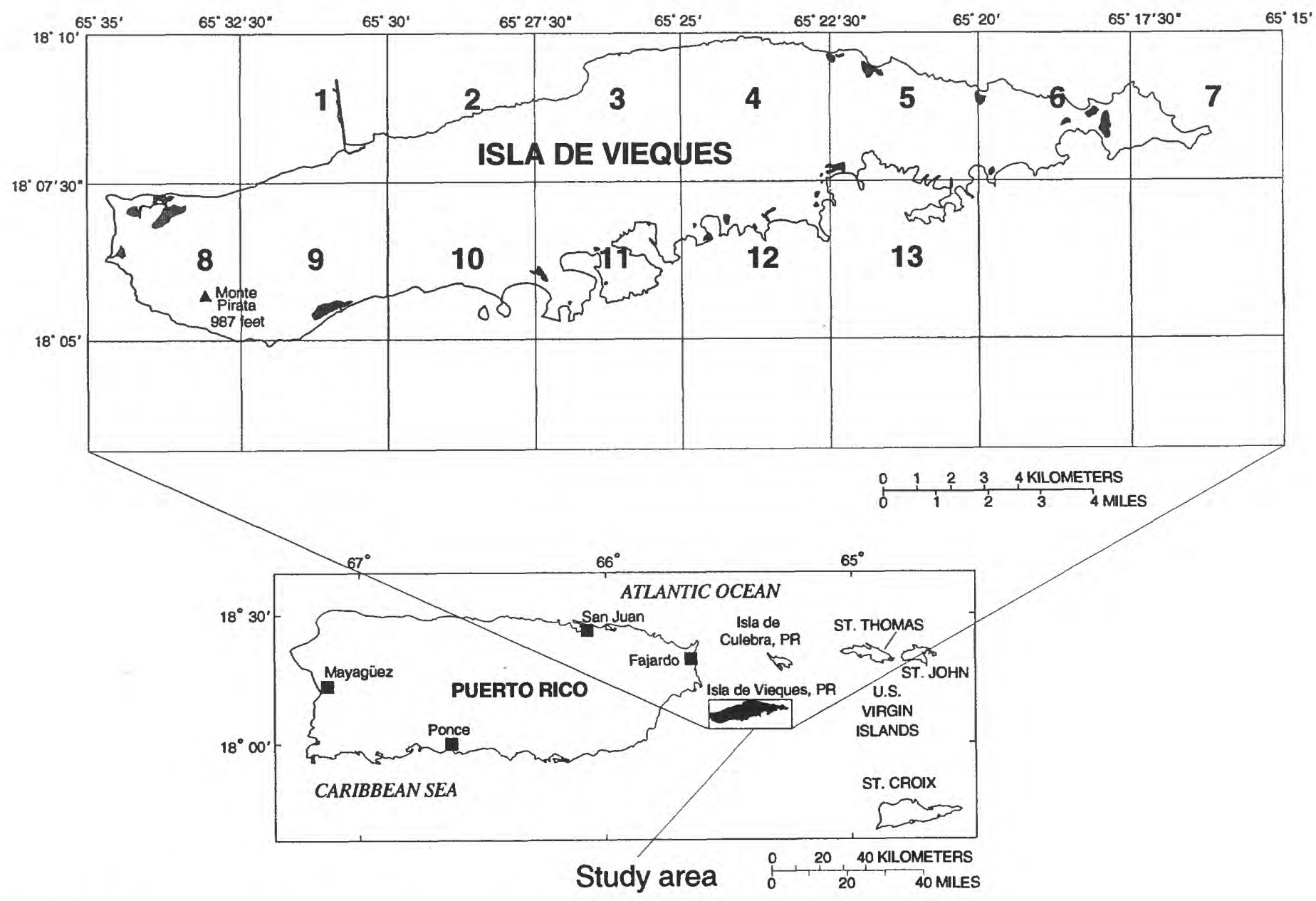

Figure 1. Location of Isla de Vieques, Puerto Rico, and area grids. 
well, the site-identification number does not change. The site-identification number is designed to correspond to the latitude-longitude coordinates of the well. Other information included in the appendix are remarks for each well.

Records of wells on Isla de Vieques were compiled from USGS GWSI records and the data were verified in the field by USGS personnel. Additional information on the water resources of the island was provided by the PRASA officials and property owners. USGS personnel performed depth of well soundings with a weighted steel tape at wells that were open at the surface. A weighted steel tape also was used to measure the depth to water below land surface.

Water samples were collected from selected wells with a bailer. The water samples were collected on the date the water-level measurement was recorded. The depth at which the water sample was collected is noted in the remarks of the appendix. If the well was pumping at the time of the survey the water sample was collected from the discharge line.

The land-surface altitude for each well location was estimated from the USGS topographic map. The contour interval of the topographic map of Isla de Vieques is 32.8 feet (10 meters). Dashed contour lines represent an interval of 3.3 feet ( 1 meter). The estimated land-surface altitudes from the USGS topographic map were converted to feet for consistency of units within the tables. The accuracy of reporting land-surface altitudes in this report is considered to be one-half of the contour interval used or 16.4 feet $(5$ meters).

Specific capacity tests were conducted, during March and April 1992, by USGS personnel for 10 PRASA wells in the Esperanza Valley. Prior to the tests, the wells were rehabilitated using compressed air to clear the perforated interval below the water table. The results from each specific capacity test is summarized in the remarks column with the measured drawdown, duration of the test in hours, the pumping rate in gallons per minute, and the date the test was conducted.

\section{REFERENCES}

Meyerhoff, H.A., 1927, Geology of the Virgin Islands, Culebra, and Vieques: Scientific Survey of Puerto Rico and the Virgin Islands, New York Academy of Sciences, v. 4, pt. II, p. 184-216.

Torres-González Sigfredo, 1989, Reconnaissance of the ground-water resources of Vieques Island, Puerto Rico: U.S. Geological Survey Water-Resources Investigations Report 86-4100, $37 \mathrm{p}$. 
THIS PAGE WAS LEFT BLANK INTENTIONALLY 


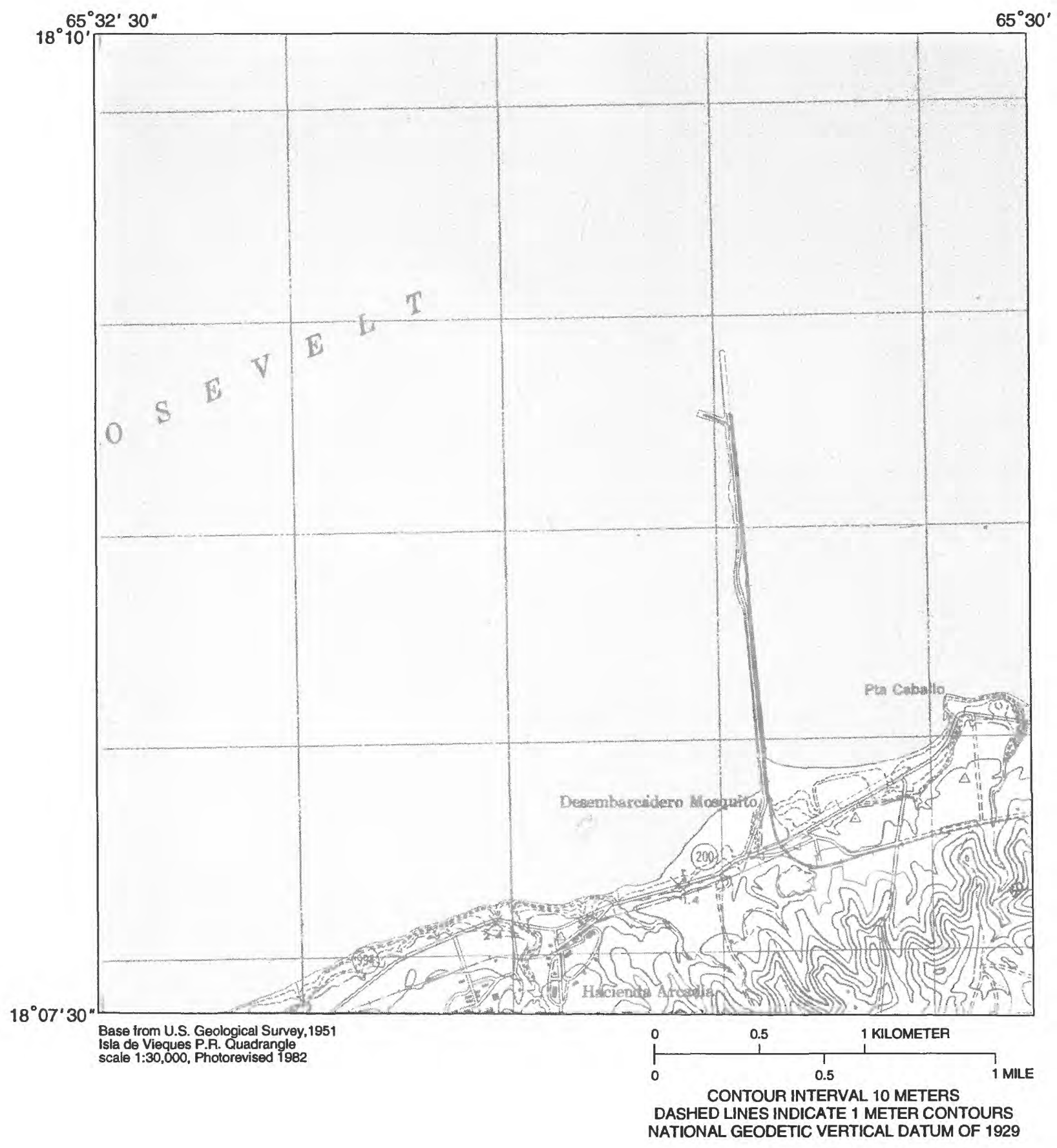

Figure 2. Location of grid 1, Isla de Vieques, Puerto Rico. 


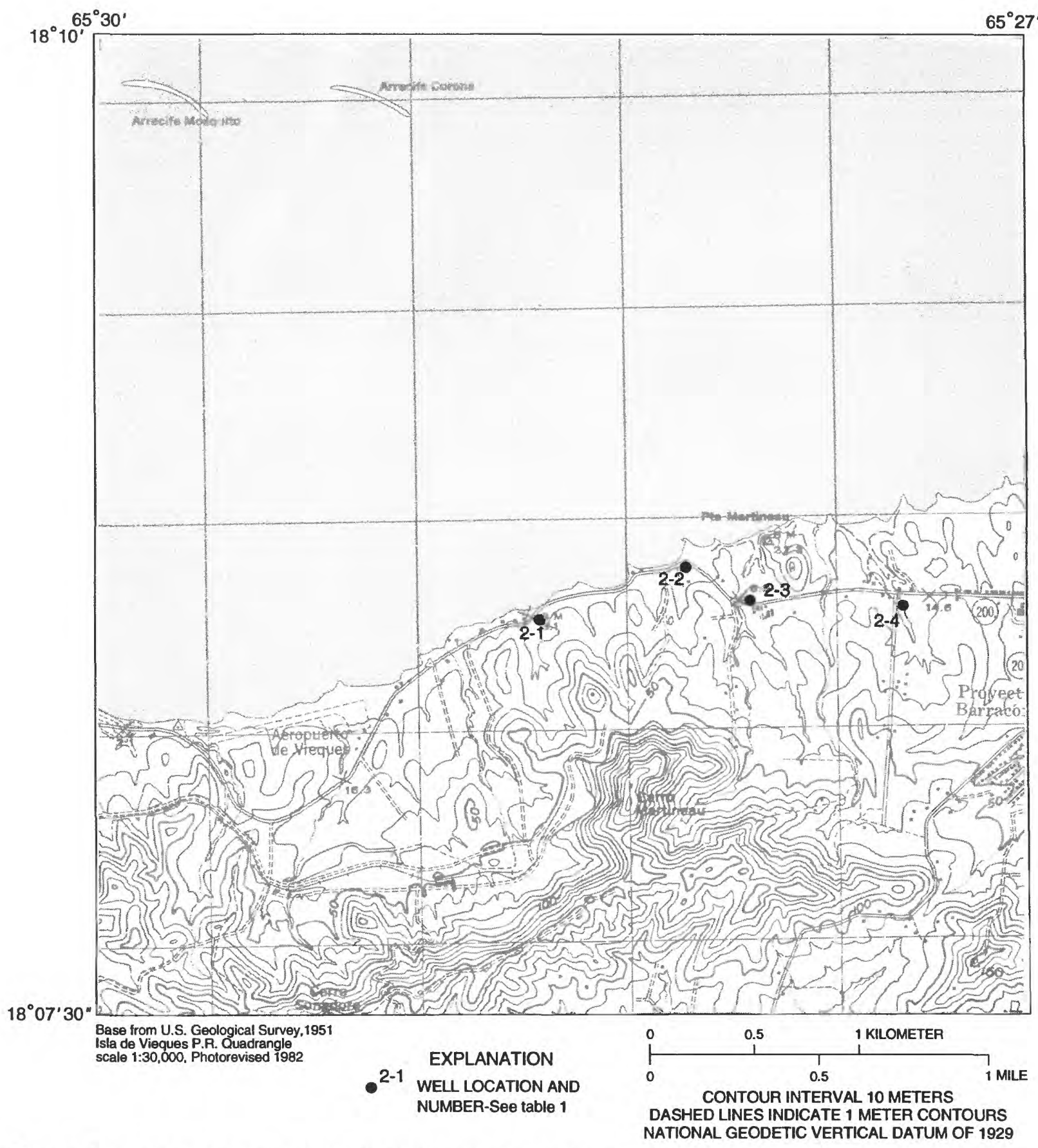

Figure 3. Location of wells in grid 2, Isla de Vieques, Puerto Rico. 
Table 1. Description of wells in grid 2, Isla de Vieques, Puerto Rico

[Location of wells shown in figure 3. Use of well: A, agriculture or stock well; NU, well not in use. $\mu \mathrm{S} / \mathrm{cm}$, microsiemen per centimeter at 25 degrees Celsius; $\mathrm{mg} / \mathrm{L}$, milligram per liter; --, no data]

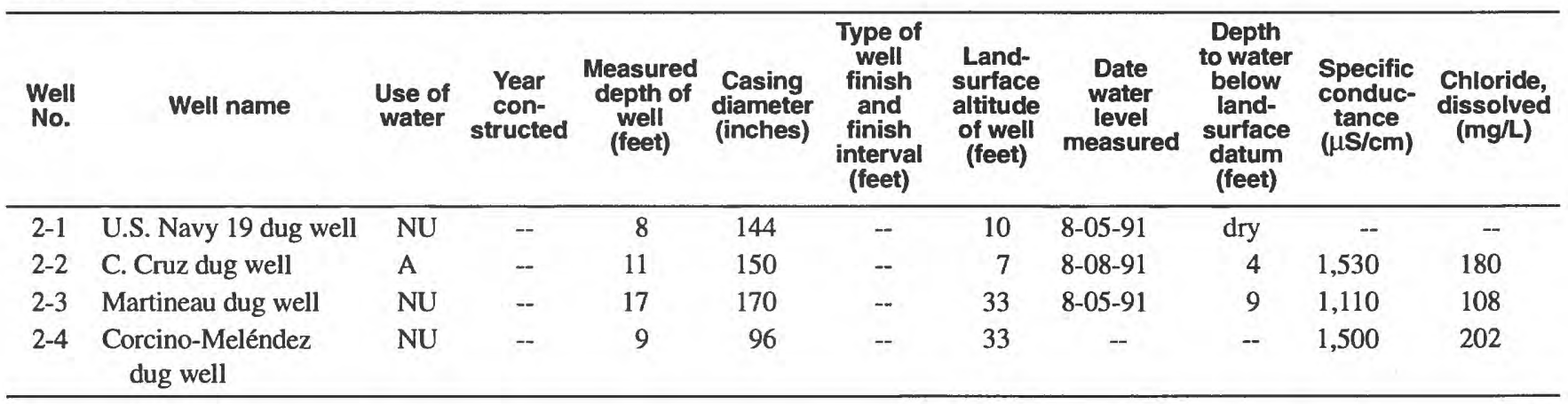




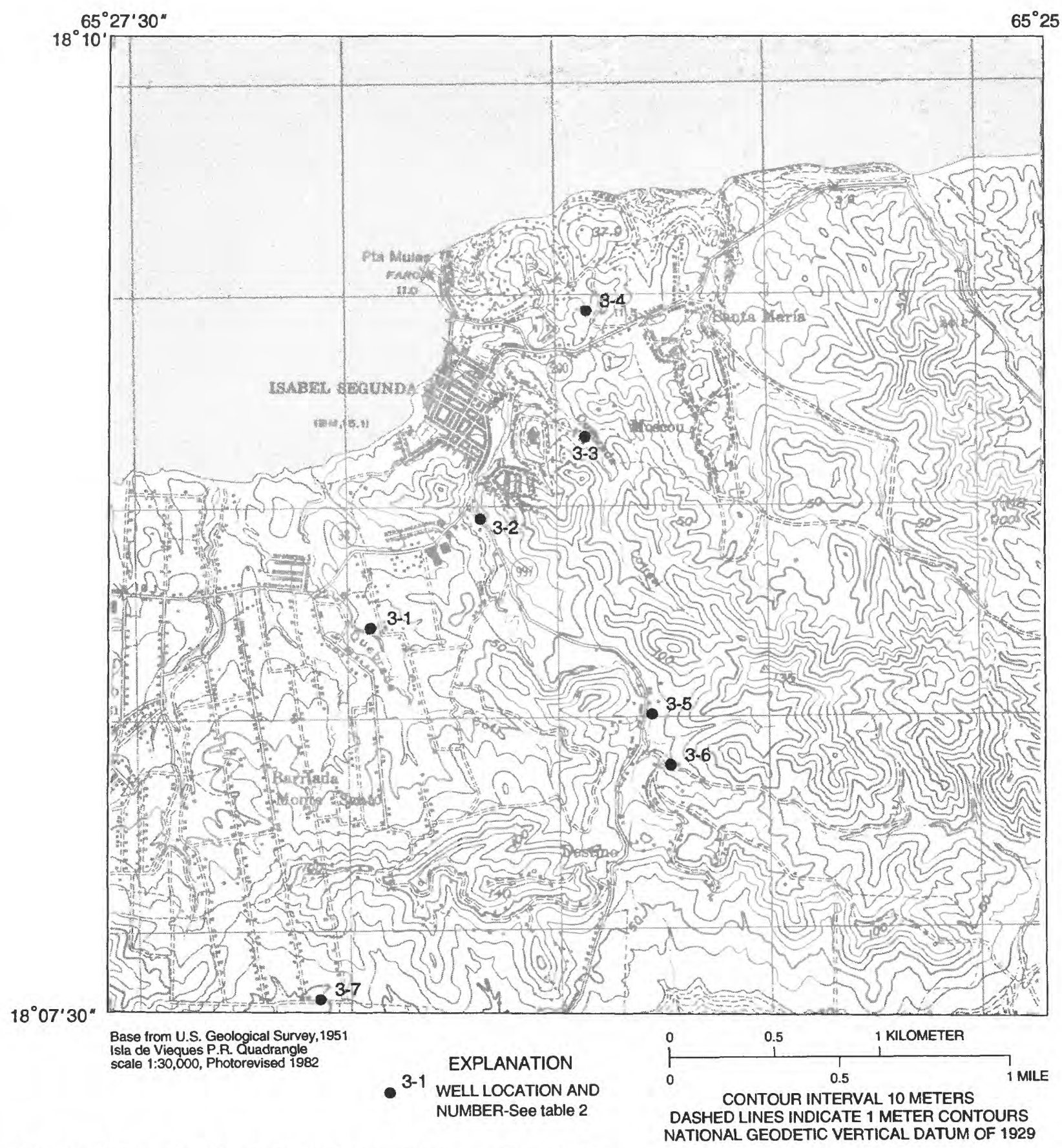

Figure 4. Location of wells in grid 3, Isla de Vieques, Puerto Rico. 
Table 2. Description of wells in grid 3 , Isla de Vieques, Puerto Rico

[Location of wells shown in figure 4. Use of water: $\mathrm{D}$, domestic well; NU, well not in use. $\mu \mathrm{S} / \mathrm{cm}$, microsiemen per centimeter at 25 degrees Celsius; mg/L, milligram per liter. --, no data]

\begin{tabular}{|c|c|c|c|c|c|c|c|c|c|c|c|}
\hline $\begin{array}{l}\text { Well } \\
\text { No. }\end{array}$ & Well name & $\begin{array}{l}\text { Use of } \\
\text { water }\end{array}$ & $\begin{array}{l}\text { Year } \\
\text { con- } \\
\text { structed }\end{array}$ & $\begin{array}{l}\text { Measured } \\
\text { depth of } \\
\text { well } \\
\text { (feet) }\end{array}$ & $\begin{array}{l}\text { Casing } \\
\text { diameter } \\
\text { (inches) }\end{array}$ & $\begin{array}{l}\text { Type of } \\
\text { well } \\
\text { finish } \\
\text { and } \\
\text { finish } \\
\text { interval } \\
\text { (feet) }\end{array}$ & $\begin{array}{l}\text { Land- } \\
\text { surface } \\
\text { altitude } \\
\text { of well } \\
\text { (feet) }\end{array}$ & $\begin{array}{c}\text { Date } \\
\text { water } \\
\text { level } \\
\text { measured }\end{array}$ & $\begin{array}{l}\text { Depth } \\
\text { to water } \\
\text { below } \\
\text { land- } \\
\text { surface } \\
\text { datum } \\
\text { (feet) }\end{array}$ & $\begin{array}{c}\text { Specific } \\
\text { conduc- } \\
\text { tance } \\
(\mu \mathrm{S} / \mathrm{cm})\end{array}$ & $\begin{array}{l}\text { Chloride, } \\
\text { dissolved } \\
\text { (mg/L) }\end{array}$ \\
\hline $3-1$ & Iglesia Metodista & $\mathrm{NU}$ & -- & 13 & 6 & -- & 115 & $8-15-91$ & 2 & -- & -- \\
\hline $3-2$ & Castaño dug well & $\mathrm{NU}$ & - & -- & 100 & -- & 66 & -- & -- & 1,640 & 178 \\
\hline $3-3$ & Metodista 1 & NU & -- & 10 & 8 & -- & 66 & $8-20-91$ & dry & -- & -- \\
\hline $3-4$ & G. Colón & $\mathrm{D}$ & - & $(1)$ & 4 & - & 82 & -- & -- & 1,540 & 176 \\
\hline $3-5$ & $\begin{array}{l}\text { Agrícola Corp. } 2 \\
\text { dug well }\end{array}$ & D & -- & 14 & 174 & - & 203 & $8-16-91$ & 4 & 1,730 & 240 \\
\hline $3-6$ & PRASA 10 dug well & NU & -- & 16 & 108 & -- & 230 & $8-19-91$ & 5 & 940 & -- \\
\hline $3-7$ & F. Peterson dug well & $\mathrm{D}$ & 1943 & 9 & 25 & - & 154 & $8-20-91$ & 2 & 1,040 & 90 \\
\hline
\end{tabular}

\footnotetext{
${ }^{1}$ See remarks in appendix 1.
} 
THIS PAGE WAS LEFT BLANK INTENTIONALLY 


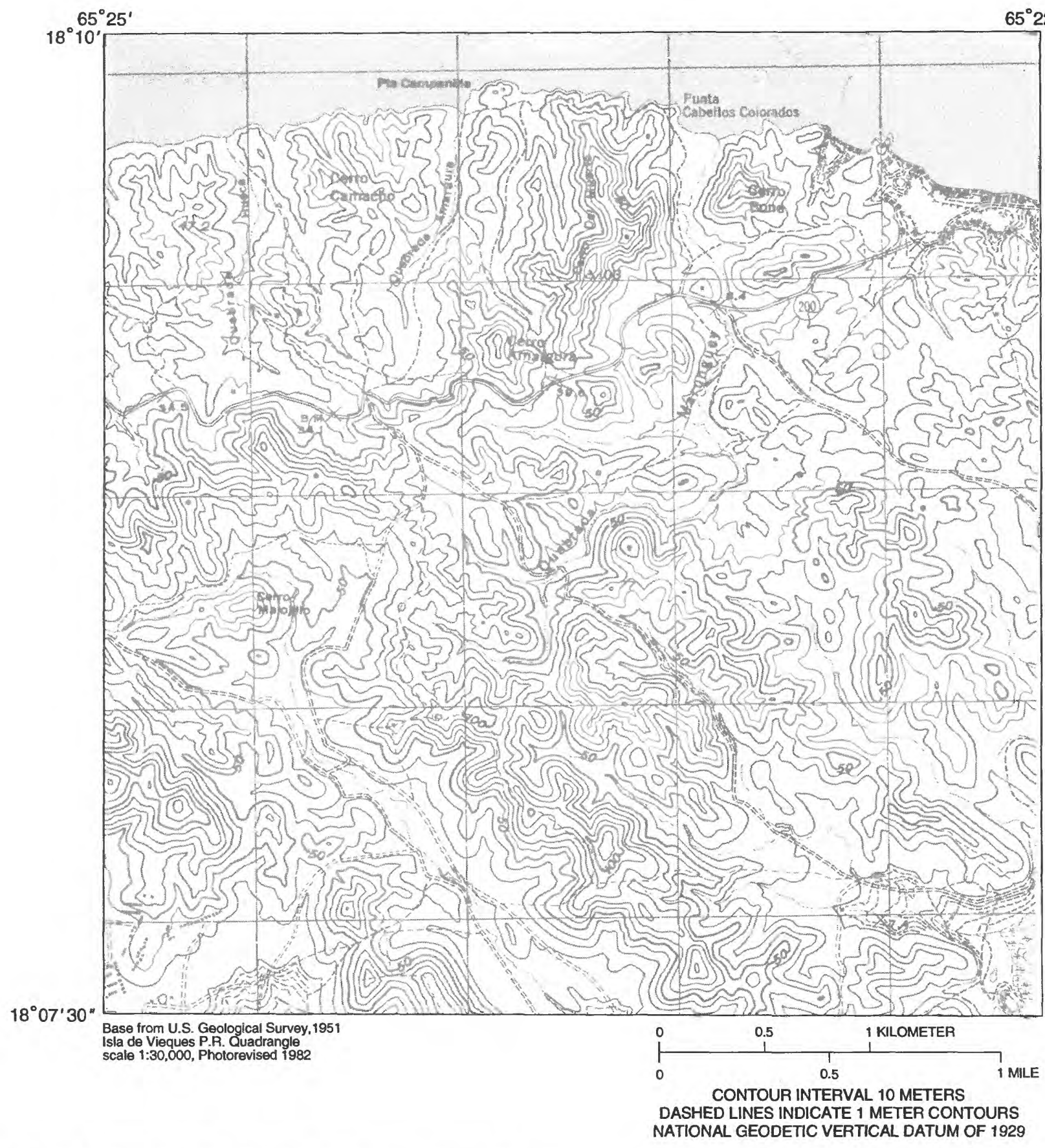

Figure 5. Location of grid 4, Isla de Vieques, Puerto Rico. 


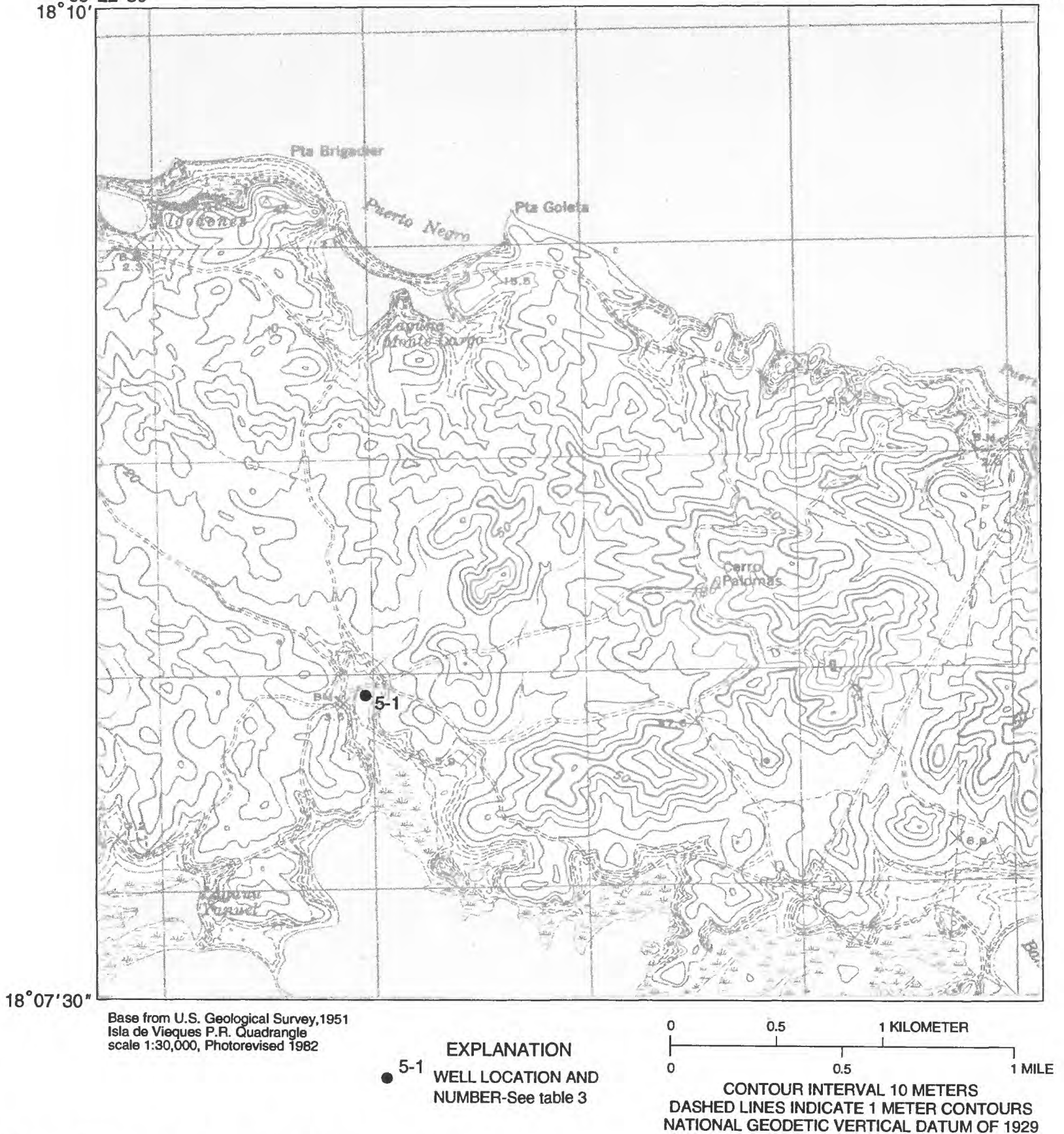

Figure 6. Location of grid 5, Isla de Vieques, Puerto Rico. 
Table 3. Description of wells in grid 5, Isla de Vieques, Puerto Rico

[Location of wells shown in figure 6. Use of water: NU, well not in use. $\mu \mathrm{S} / \mathrm{cm}$, microsiemen per centimeter at 25 degrees Celsius; mg/L, milligram per liter. ,-- no data]

\begin{tabular}{|c|c|c|c|c|c|c|c|c|c|c|c|}
\hline $\begin{array}{l}\text { Well } \\
\text { No. }\end{array}$ & Well name & $\begin{array}{l}\text { Use of } \\
\text { water }\end{array}$ & $\begin{array}{c}\text { Year } \\
\text { con- } \\
\text { structed }\end{array}$ & $\begin{array}{l}\text { Measured } \\
\text { depth of } \\
\text { well } \\
\text { (feet) }\end{array}$ & $\begin{array}{c}\text { Casing } \\
\text { diameter } \\
\text { (inches) }\end{array}$ & $\begin{array}{l}\text { Type of } \\
\text { well } \\
\text { finish } \\
\text { and } \\
\text { finish } \\
\text { interval } \\
\text { (feet) }\end{array}$ & $\begin{array}{l}\text { Land- } \\
\text { surface } \\
\text { altitude } \\
\text { of well } \\
\text { (feet) }\end{array}$ & $\begin{array}{l}\text { Date } \\
\text { water } \\
\text { level } \\
\text { measured }\end{array}$ & $\begin{array}{l}\text { Depth to } \\
\text { water } \\
\text { below } \\
\text { land- } \\
\text { surface } \\
\text { datum } \\
\text { (feet) }\end{array}$ & $\begin{array}{c}\text { Specific } \\
\text { conduc- } \\
\text { tance } \\
(\mu S / \mathrm{cm})\end{array}$ & $\begin{array}{l}\text { Chloride, } \\
\text { dissolved } \\
\text { (mg/L) }\end{array}$ \\
\hline $5-1$ & Camp García dug well & $\mathrm{NU}$ & -- & 13 & 120 & - & 20 & 10 & $8-27-91$ & 13,670 & 4,138 \\
\hline
\end{tabular}






Figure 7. Location of grid 6, Isla de Vieques, Puerto Rico. 


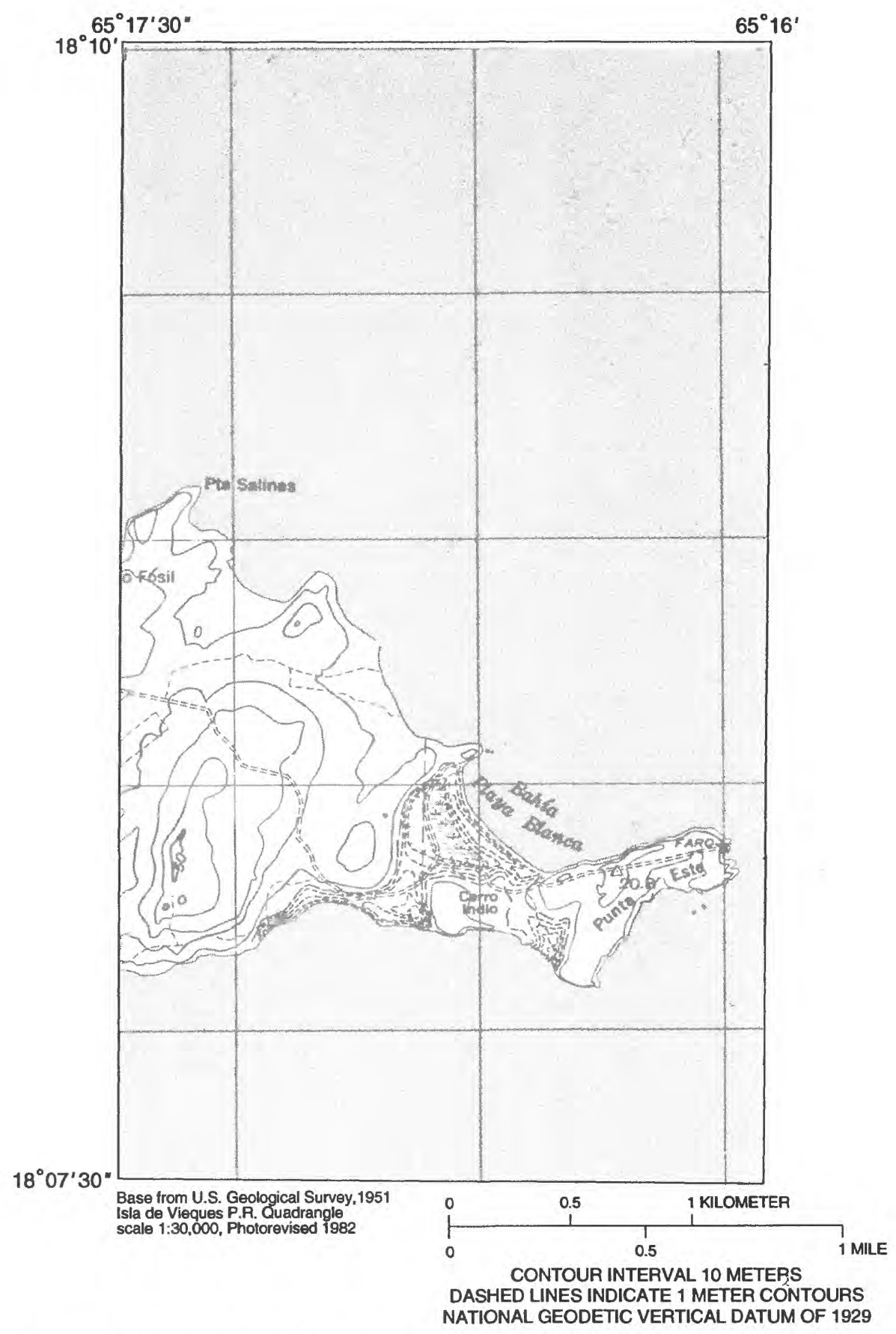

Figure 8. Location of grid 7, Isla de Vieques, Puerto Rico. 
THIS PAGE WAS EEFT BLANK INTENTIONALLY 


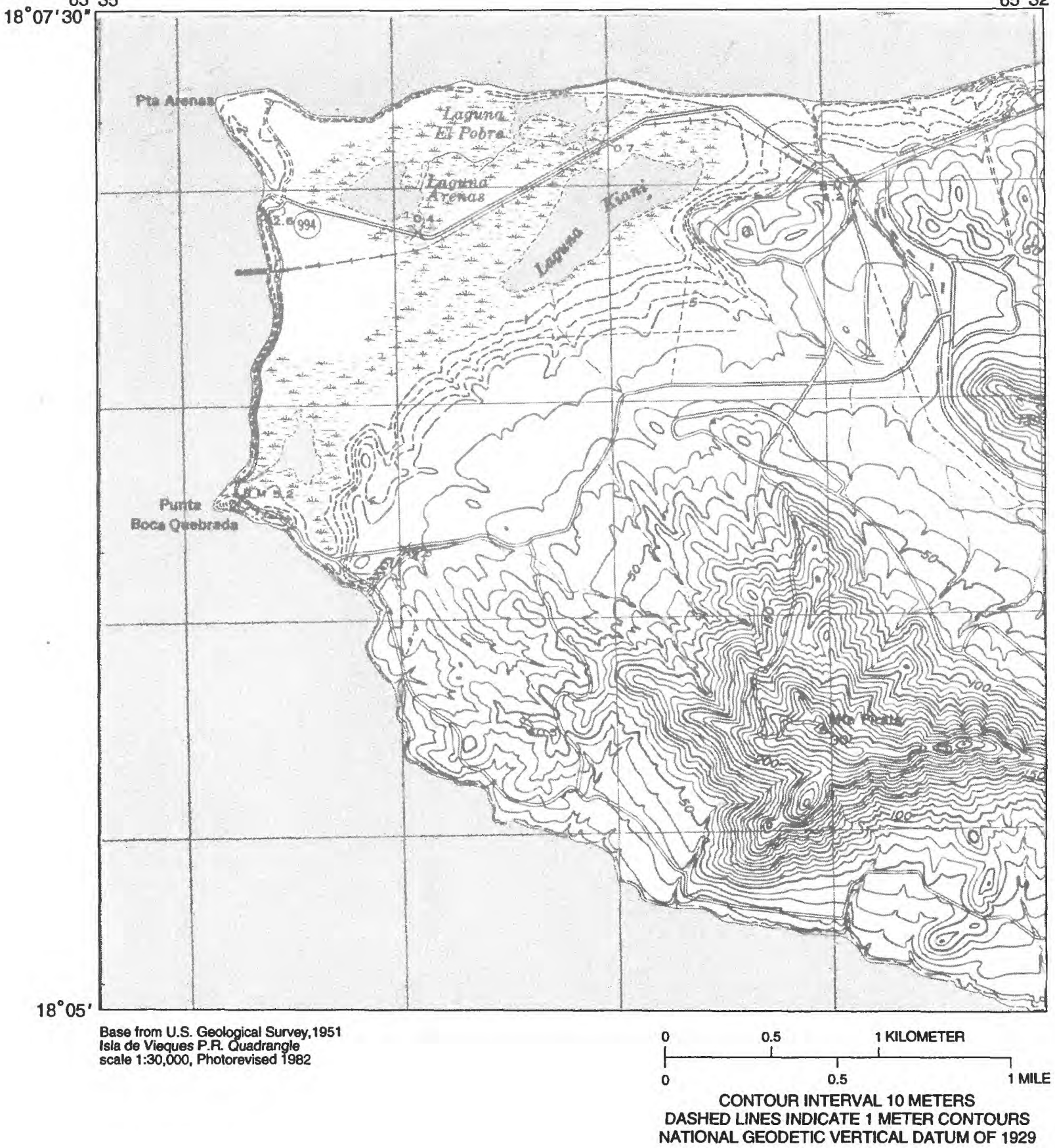

Figure 9. Location of grid 8, Isla de Vieques, Puerto Rico. 


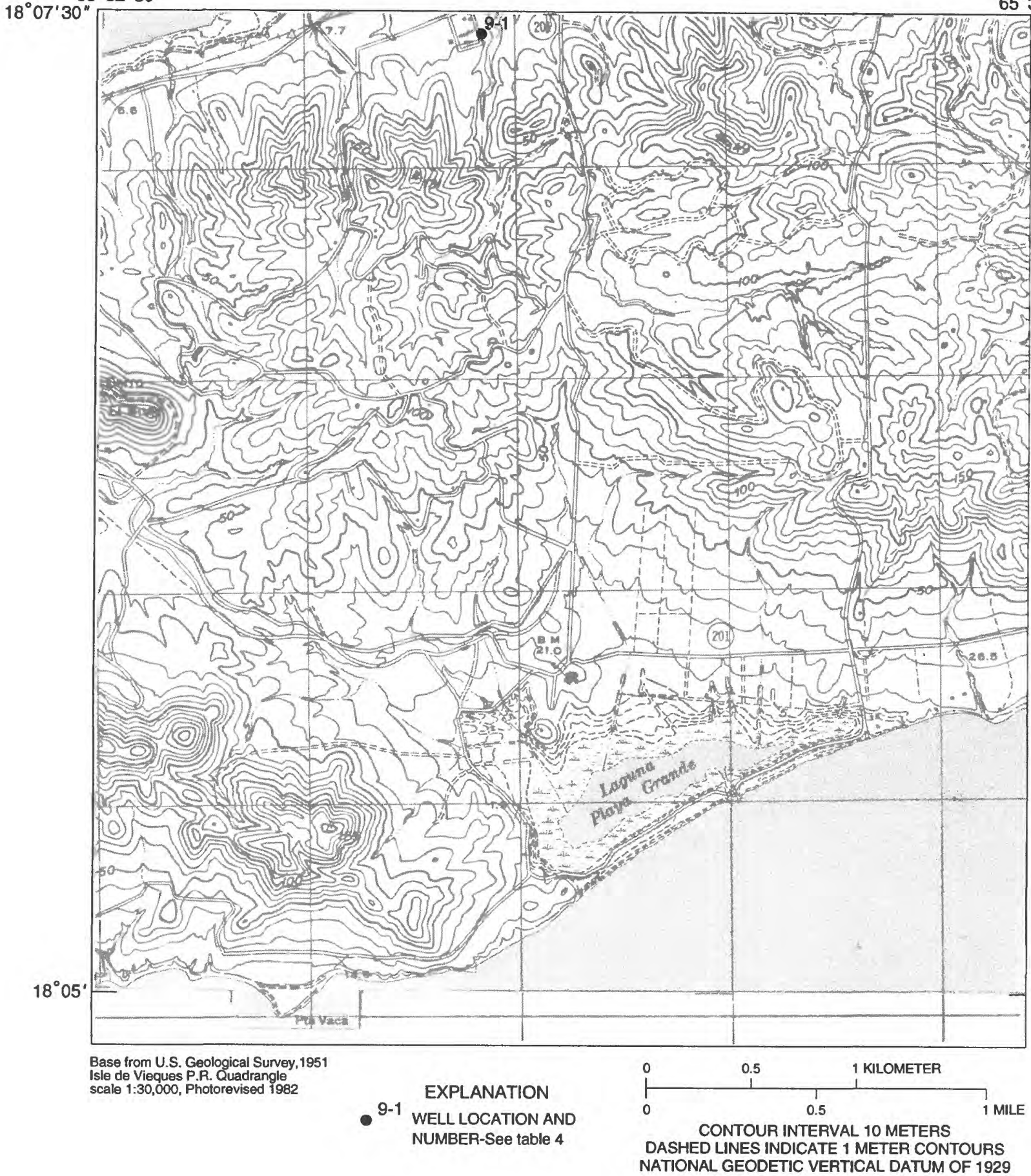

Figure 10. Location of grid 9, Isla de Vieques, Puerto Rico. 
Table 4. Description of well in grid 9, Isla de Vieques, Puerto Rico

[Location of well shown in figure 10. Use of water: $\mathrm{NU}$, well not in use. $\mu \mathrm{S} / \mathrm{cm}$, microsiemen per centimeter at 25 degrees Celsius; $\mathrm{mg} / \mathrm{L}$, milligram per liter. ,-- no data]

\begin{tabular}{|c|c|c|c|c|c|c|c|c|c|c|c|}
\hline $\begin{array}{l}\text { Well } \\
\text { No. }\end{array}$ & Well name & $\begin{array}{l}\text { Use of } \\
\text { water }\end{array}$ & $\begin{array}{c}\text { Year } \\
\text { con- } \\
\text { structed }\end{array}$ & $\begin{array}{l}\text { Measured } \\
\text { depth of } \\
\text { well } \\
\text { (feet) }\end{array}$ & $\begin{array}{l}\text { Casing } \\
\text { diameter } \\
\text { (inches) }\end{array}$ & $\begin{array}{l}\text { Type of } \\
\text { well } \\
\text { finish } \\
\text { and } \\
\text { finish } \\
\text { interval } \\
\text { (feet) }\end{array}$ & $\begin{array}{l}\text { Land- } \\
\text { surface } \\
\text { altitude } \\
\text { of well } \\
\text { (feet) }\end{array}$ & $\begin{array}{c}\text { Date } \\
\text { water } \\
\text { level } \\
\text { measured }\end{array}$ & $\begin{array}{l}\text { Depth to } \\
\text { water } \\
\text { below } \\
\text { land- } \\
\text { surface } \\
\text { datum } \\
\text { (feet) }\end{array}$ & $\begin{array}{c}\text { Specific } \\
\text { conduc- } \\
\text { tance } \\
(\mu \mathrm{S} / \mathrm{cm})\end{array}$ & $\begin{array}{l}\text { Chloride, } \\
\text { dissolved } \\
\text { (mg/L) }\end{array}$ \\
\hline $9-1$ & U.S. Navy 17 & $\mathrm{NU}$ & - & 69 & 8 & - & 59 & $8-21-91$ & 51 & 1,230 & 200 \\
\hline
\end{tabular}




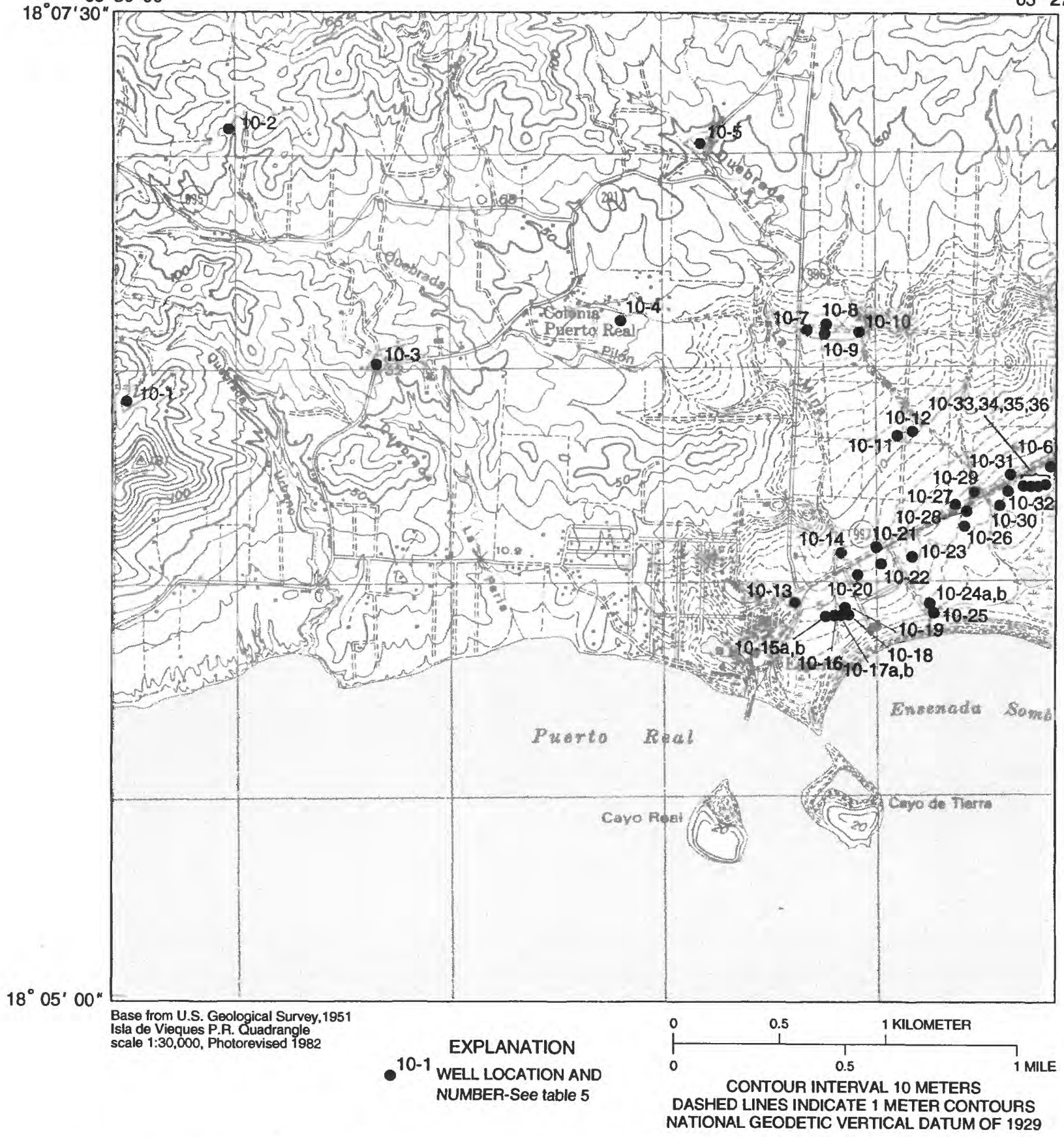

Figure 11. Location of wells in grid 10, Isla de Vieques, Puerto Rico. 
Table 5. Description of wells in grid 10, Isla de Vieques, Puerto Rico

[Location of wells shown in figure 11. Use of water: D, domestic well; NU, well not in use. Type of well: P, perforated; $S$, screened. $\mu$ S/cm, microsiemen per centimeter at 25 degrees Celsius; $\mathrm{mg} / \mathrm{L}$, milligram per liter. --, no data]

\begin{tabular}{|c|c|c|c|c|c|c|c|c|c|c|c|}
\hline $\begin{array}{l}\text { Well } \\
\text { No. }\end{array}$ & Well name & $\begin{array}{l}\text { Use of } \\
\text { water }\end{array}$ & $\begin{array}{c}\text { Year } \\
\text { con- } \\
\text { structed }\end{array}$ & $\begin{array}{l}\text { Measured } \\
\text { depth of } \\
\text { well } \\
\text { (feet) }\end{array}$ & $\begin{array}{l}\text { Casing } \\
\text { diameter } \\
\text { (inches) }\end{array}$ & $\begin{array}{l}\text { Type of } \\
\text { well } \\
\text { finish } \\
\text { and } \\
\text { finish } \\
\text { interval } \\
\text { (feet) }\end{array}$ & $\begin{array}{l}\text { Land- } \\
\text { surface } \\
\text { altitude } \\
\text { of well } \\
\text { (feet) }\end{array}$ & $\begin{array}{c}\text { Date } \\
\text { water } \\
\text { level } \\
\text { measured }\end{array}$ & $\begin{array}{l}\text { Depth } \\
\text { to water } \\
\text { below } \\
\text { land- } \\
\text { surface } \\
\text { datum } \\
\text { (feet) }\end{array}$ & $\begin{array}{c}\text { Specific } \\
\text { conduc- } \\
\text { tance } \\
(\mu \mathrm{S} / \mathrm{cm})\end{array}$ & $\begin{array}{l}\text { Chloride, } \\
\text { dissolved } \\
\text { (mg/L) }\end{array}$ \\
\hline $10-1$ & Pilón 1 dug well & $\mathrm{NU}$ & - & 9 & ${ }^{1} 6$ & - & 292 & $8-29-91$ & 4 & 600 & 78 \\
\hline $10-2$ & $\begin{array}{l}\text { Sucn Díaz } 1 \text { dug } \\
\text { well }\end{array}$ & D & -- & 14 & 70 & -- & 295 & 8-29-91 & 6 & 1,170 & 156 \\
\hline $10-3$ & O. González & NU & -- & - & 4 & -- & 105 & - & - & 950 & 104 \\
\hline $10-4$ & Vieques 12 & NU & 1986 & 57 & 4 & S 23-38 & 105 & 8-01-91 & 43 & 1,080 & 90 \\
\hline $10-5$ & $\begin{array}{l}\text { U.S. Navy } 15 \text { dug } \\
\text { well }\end{array}$ & $\mathrm{NU}$ & -- & -- & 26 & -- & 148 & 8-03-91 & dry & -- & -- \\
\hline $10-6$ & Vieques 8 & NU & 1986 & 32 & 4 & S $28-32$ & 23 & $8-05-91$ & 6 & 1,420 & 178 \\
\hline $10-7$ & PRASA 1 & NU & -- & 34 & 10 & -- & 66 & $8-08-91$ & dry & -- & -- \\
\hline $10-8$ & USGS La Mina & NU & -- & 48 & 4 & -- & 70 & $8-08-91$ & 34 & 800 & 68 \\
\hline $10-9$ & PRASA 2 & NU & -- & 26 & 10 & -- & 69 & $8-08-91$ & dry & -- & -- \\
\hline $10-10$ & PRASA 3 & NU & -- & 26 & 10 & -- & 66 & $8-08-91$ & dry & -- & -- \\
\hline $10-11$ & La Mina 1 & NU & -- & 21 & 4 & -- & 36 & $8-12-91$ & dry & -- & -- \\
\hline $10-12$ & La Mina 2 & NU & -- & 46 & 4 & - & 36 & $8-12-91$ & 22 & 990 & 112 \\
\hline $10-13$ & Fesa 2 dug well & NU & -- & 21 & 156 & -- & 10 & $8-02-91$ & 13 & 1,650 & 196 \\
\hline $10-14$ & O'Conner 12 & NU & -- & 32 & 12 & -- & 36 & $8-05-91$ & 15 & 930 & 118 \\
\hline $10-15 a$ & PRASA A-5 & NU & -- & $\left({ }^{1}\right)$ & 7 & -- & 16 & -- & -- & -- & -- \\
\hline $10-15 b$ & PRASA A-5A & NU & -- & 34 & 10 & -- & 16 & $8-02-91$ & 11 & 950 & 126 \\
\hline $10-16$ & PRASA A-4 & NU & -- & $\left({ }^{1}\right)$ & 10 & - & 7 & -- & -- & -- & - \\
\hline $10-17 a$ & PRASA A-2 & NU & 1966 & 40 & 10 & P $25-40$ & 16 & $3-26-92$ & 8 & 820 & 92 \\
\hline $10-17 b$ & PRASA A-3 & NU & 1966 & 48 & 10 & P $25-48$ & 16 & $3-19-92$ & 9 & 830 & 90 \\
\hline $10-18$ & PRASA A-1 & NU & 1966 & 44 & 10 & P 20-44 & 16 & $3-11-92$ & 9 & 710 & 80 \\
\hline $10-19$ & USGS-piezometer 1 & NU & -- & 25 & 2 & -- & 16 & $8-29-91$ & 10 & 700 & 96 \\
\hline $10-20$ & Vieques $5 \mathrm{~A}$ & NU & 1986 & 47 & 4 & S 27-42 & 36 & $8-01-91$ & 10 & 400 & 40 \\
\hline $10-21$ & PRASA 4 & NU & -- & 24 & 8 & -- & 36 & $8-02-91$ & 14 & 960 & 100 \\
\hline $10-22$ & Vieques 5 & NU & 1986 & 37 & 4 & S 26-41 & 36 & 8-01-91 & 11 & 890 & 90 \\
\hline $10-23$ & PRASA D-3 & NU & 1966 & 46 & 10 & -- & 32 & $3-26-92$ & 10 & 960 & 90 \\
\hline $10-24 a$ & PRIDCO 9A & NU & -- & ${ }^{17}$ & 4 & -- & 21 & $7-31-91$ & 6 & 2,320 & 294 \\
\hline $10-24 b$ & PRIDCO 9 & $\mathrm{NU}$ & -- & 83 & 4 & -- & 21 & $7-31-91$ & 4 & 1,080 & 140 \\
\hline $10-25$ & PRASA 151 & $\mathrm{NU}$ & -- & 48 & 6 & -- & 20 & $7-31-91$ & 2 & 370 & 70 \\
\hline $10-26$ & PRASA D-2 & $\mathrm{NU}$ & 1966 & 66 & 10 & -- & 26 & $4-01-92$ & 8 & 975 & 100 \\
\hline $10-27$ & PRASA 5 & $\mathrm{NU}$ & - & 27 & 10 & -- & 30 & $8-06-91$ & 8 & - & -- \\
\hline $10-28$ & Vieques 18 & NU & 1986 & 40 & 4 & S $28-43$ & 26 & $8-14-91$ & 8 & 590 & 64 \\
\hline $10-29$ & PRASA 6 & NU & -- & 29 & 10 & -- & 26 & 8-06-91 & 8 & 550 & 84 \\
\hline $10-30$ & PRASA D-1 & NU & 1966 & 74 & 10 & -- & 23 & $4-01-92$ & 6 & 1,100 & 120 \\
\hline $10-31$ & PRASA 7 & NU & -- & 11 & 10 & -- & 23 & $8-06-91$ & 8 & 1,750 & 146 \\
\hline $10-32$ & Vieques 22 & NU & 1986 & 46 & 4 & S 33-47 & 36 & $8-05-91$ & 6 & 1,050 & 124 \\
\hline $10-33$ & PRASA B-4 & NU & 1966 & 45 & 10 & P $15-45$ & 23 & 4-02-92 & 4 & 990 & 90 \\
\hline $10-34$ & PRASA B-3 & NU & 1966 & 47 & 10 & P 21-47 & 23 & $3-13-92$ & 3 & 1,030 & 120 \\
\hline $10-35$ & PRASA B-2 & NU & 1966 & 50 & 10 & P 20-50 & 23 & 4-03-92 & 4 & 1,100 & 110 \\
\hline $10-36$ & PRASA B-1 & NU & 1966 & 62 & 10 & P 20-57 & 23 & 4-02-92 & 4 & 1,210 & 120 \\
\hline
\end{tabular}

\footnotetext{
${ }^{1}$ See remarks in appendix.
} 


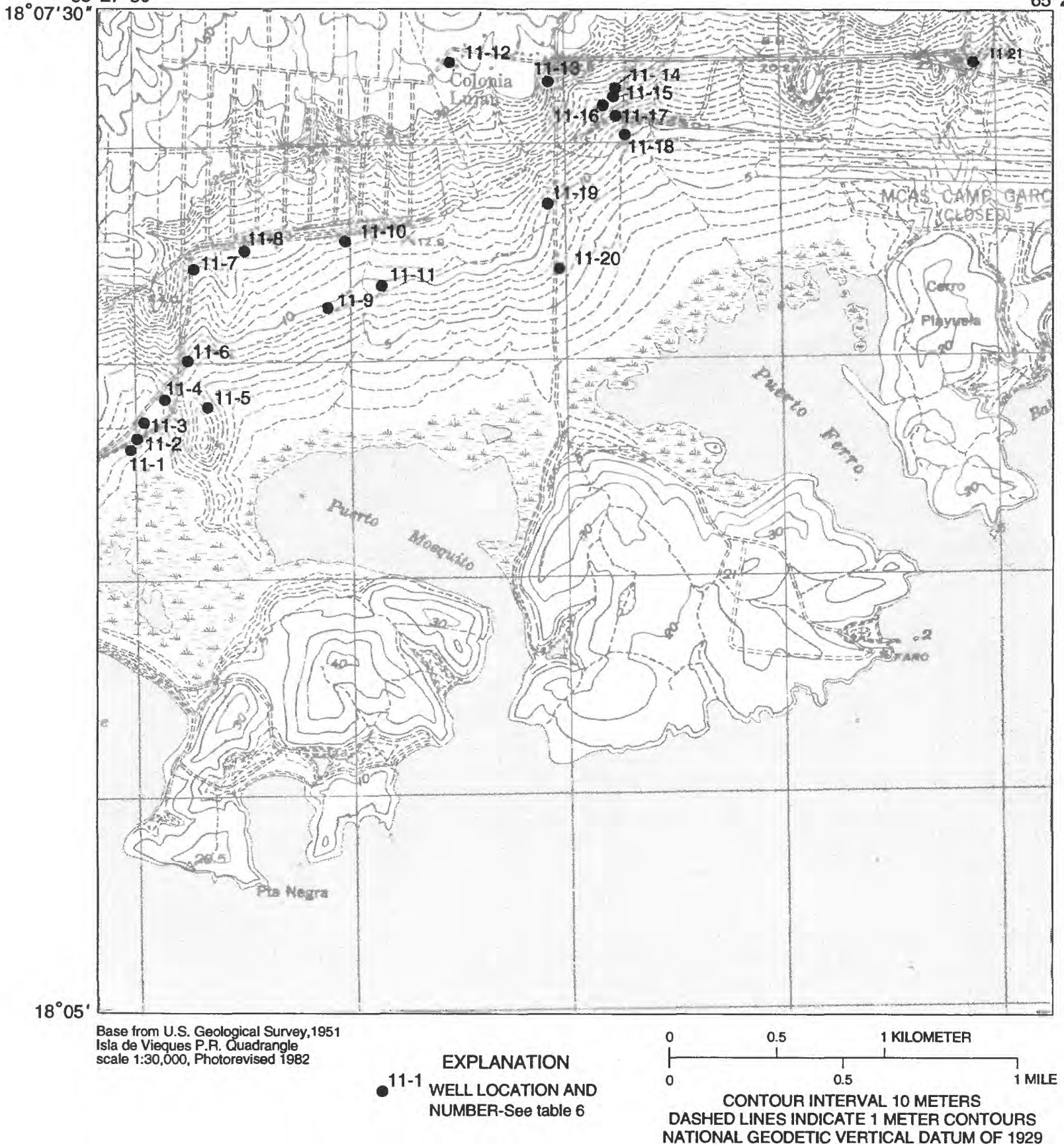

Figure 12. Location of wells in grid 11, Isla de Vieques, Puerto Rico. 
Table 6. Description of wells in grid 11, Isla de Vieques, Puerto Rico

[Location of wells shown in figure 12. Use of water: PS, public-supply well; NU, well not in use. $\mu \mathrm{S} / \mathrm{cm}$, microsiemen per centimeter at 25 degrees Celsius; $\mathrm{mg} / \mathrm{L}$, milligram per liter. --, no data]

\begin{tabular}{|c|c|c|c|c|c|c|c|c|c|c|c|}
\hline $\begin{array}{l}\text { Well } \\
\text { No. }\end{array}$ & Well name & $\begin{array}{l}\text { Use of } \\
\text { water }\end{array}$ & $\begin{array}{c}\text { Year } \\
\text { con- } \\
\text { structed }\end{array}$ & $\begin{array}{c}\text { Measured } \\
\text { depth of } \\
\text { well } \\
\text { (feet) }\end{array}$ & $\begin{array}{l}\text { Casing } \\
\text { diameter } \\
\text { (inches) }\end{array}$ & $\begin{array}{l}\text { Type of } \\
\text { well } \\
\text { finish } \\
\text { and } \\
\text { finish } \\
\text { interval } \\
\text { (feet) }\end{array}$ & $\begin{array}{l}\text { Land- } \\
\text { surface } \\
\text { altitude } \\
\text { of well } \\
\text { (feet) }\end{array}$ & $\begin{array}{c}\text { Date } \\
\text { water } \\
\text { level } \\
\text { measured }\end{array}$ & $\begin{array}{c}\text { Depth } \\
\text { to } \\
\text { water } \\
\text { below } \\
\text { land- } \\
\text { surface } \\
\text { datum } \\
\text { (feet) }\end{array}$ & $\begin{array}{c}\text { Specific } \\
\text { conduc- } \\
\text { tance } \\
(\mu \mathrm{S} / \mathrm{cm})\end{array}$ & $\begin{array}{l}\text { Chloride, } \\
\text { dissolved } \\
\text { (mg/L) }\end{array}$ \\
\hline $11-1$ & PRASA C-3 & $\mathrm{NU}$ & 1966 & $T_{34}$ & 10 & -- & 23 & $8-07-91$ & 6 & 200 & 24 \\
\hline $11-2$ & PRASA C-2 & NU & 1966 & 49 & 10 & P 25-49 & 23 & 8-07-91 & 7 & 1,100 & 166 \\
\hline $11-3$ & PRASA C-1 & NU & 1966 & 44 & 10 & P 18-44 & 24 & 8-07-91 & 6 & 430 & 22 \\
\hline $11-4$ & Vieques 14 & NU & 1986 & ${ }^{2} 48$ & 4 & S 41-52 & 20 & 8-07-91 & 17 & -- & - \\
\hline $11-5$ & Vieques 16 & NU & 1986 & 42 & 4 & S 38-43 & 18 & 8-07-91 & 26 & 2,840 & 782 \\
\hline $11-6$ & Vieques 17 & NU & 1986 & -- & 4 & S 26-36 & 25 & -- & -- & -- & - \\
\hline $11-7$ & Vieques 15 & $\mathrm{NU}$ & 1986 & 49 & 4 & S 42-52 & 52 & 8-13-91 & 31 & 1,010 & 74 \\
\hline $11-8$ & Vieques 8A & $\mathrm{NU}$ & 1986 & -- & 4 & S 33-43 & 56 & -- & -- & 410 & -- \\
\hline $11-9$ & O'Conner 4 & $\mathrm{NU}$ & - & 40 & 6 & - & 33 & 8-13-91 & 29 & 1,180 & 104 \\
\hline $11-10$ & Vieques 1 & $\mathrm{NU}$ & 1986 & 50 & 4 & S $32-52$ & 46 & $9-06-91$ & 12 & 1,200 & 100 \\
\hline $11-11$ & O'Conner 5 & $\mathrm{NU}$ & -- & 21 & 10 & -- & 33 & $10-19-91$ & dry & -- & -- \\
\hline $11-12$ & $\begin{array}{l}\text { Land Authority dug } \\
\text { well }\end{array}$ & $\mathrm{NU}$ & - & 12 & 132 & - & 148 & $8-20-91$ & 8 & 1,030 & 76 \\
\hline $11-13$ & Vieques 19 & NU & 1986 & $1_{46}$ & 4 & S 48-58 & 86 & $8-13-91$ & 43 & 1,530 & 110 \\
\hline $11-14$ & U.S. Marines 6 & PS & - & - & 10 & -- & 66 & -- & -- & -- & -- \\
\hline $11-15$ & U.S. Navy 13 & NU & -- & $\left({ }^{1}\right)$ & 10 & - & 56 & - & -- & -- & -- \\
\hline $11-16$ & $\begin{array}{l}\text { U.S. Navy Camp } \\
\text { García }\end{array}$ & NU & -- & 28 & 6 & - & 69 & $8-23-91$ & 21 & 940 & 92 \\
\hline $11-17$ & U.S. Navy 14 & PS & -- & -- & 10 & -- & 44 & -- & -- & -- & -- \\
\hline $11-18$ & USGS-piezometer 2 & NU & -- & 25 & 2 & - & 49 & $8-22-91$ & dry & - & - \\
\hline $11-19$ & Vieques 2 & NU & -- & 54 & 4 & -- & 49 & 8-13-91 & 17 & 1,330 & 110 \\
\hline $11-20$ & Vieques 4 & NU & 1986 & ${ }^{1} 29$ & 4 & S $35-45$ & 20 & $8-21-91$ & 28 & 500 & 46 \\
\hline $11-21$ & U.S. Marines 4 & NU & 1965 & 59 & 8 & -- & 49 & $8-22-91$ & 31 & 1,750 & 138 \\
\hline
\end{tabular}

${ }^{1}$ See remarks in appendix 1 .

${ }^{2}$ Reported depth. 


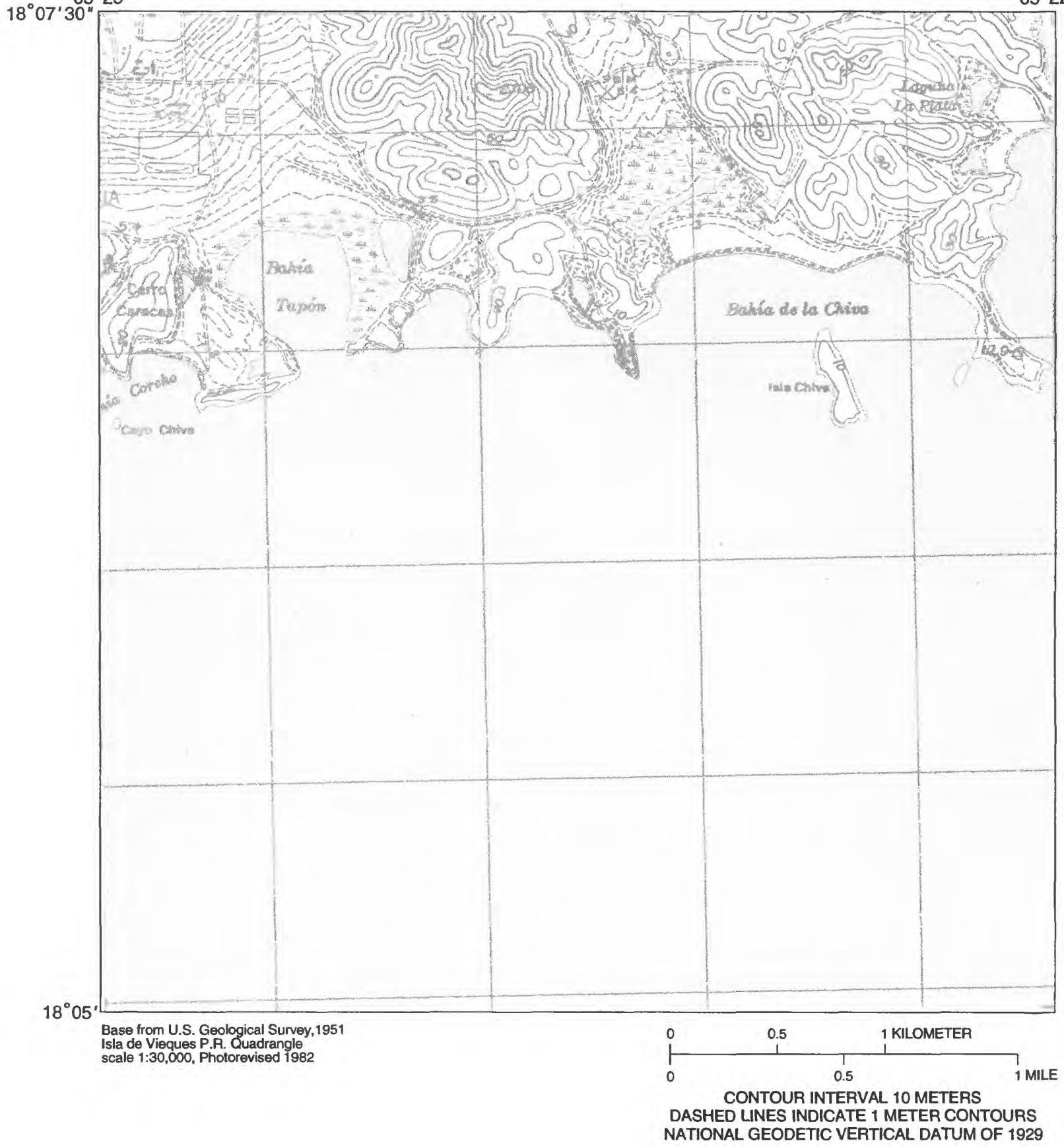

Figure 13. Location of grid 12, Isla de Vieques, Puerto Rico. 


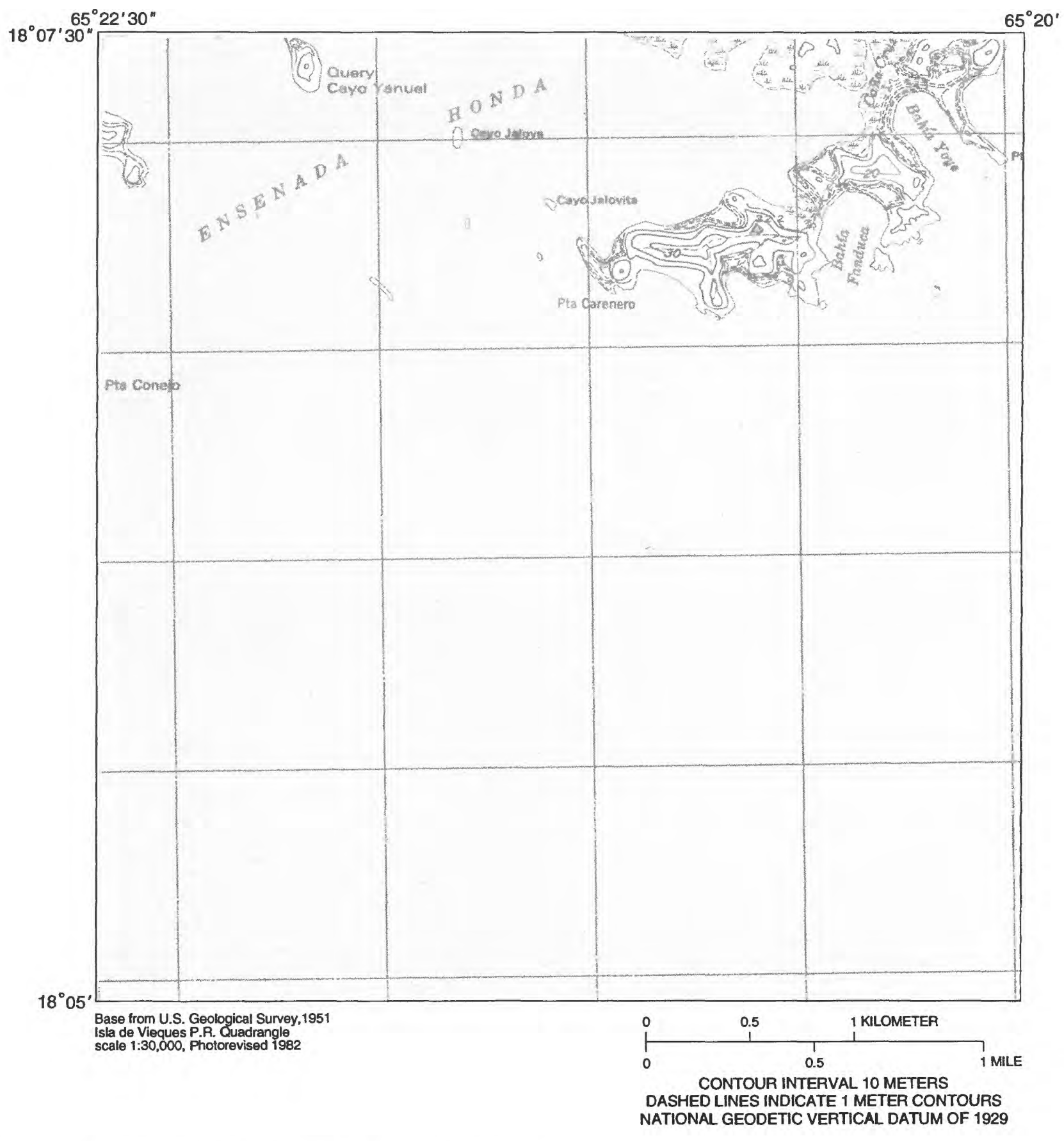

Figure 14. Location of grid 13, Isla de Vieques, Puerto Rico. 
THIS PAGE WAS LEFT BLANK INTENTIONALLY 
APPENDIX 1

27 
THIS PAGE WAS LEFT BLANK

INTENTIONALLY

28 
Appendix 1. Well names, site-identification number, and remarks for wells on Isla de Vieques, Puerto Rico

[Site identification No.: Unique number for each site based on the latitude and longitude of the site. First six digits are latitude, next seven digits are longitude, and final two digits are a sequence number to uniquely identify each site. --, no data]

\begin{tabular}{lcccc}
$\begin{array}{l}\text { Well } \\
\text { No. }\end{array}$ & $\begin{array}{c}\text { Figure } \\
\text { No. }\end{array}$ & Well name & Site identification No. & Remarks \\
\hline
\end{tabular}

$\begin{array}{lll}2-1 & 3 & \text { U.S. Navy } 19 \text { dug well } \\ 2-2 & 3 & \text { C. Cruz dug well }\end{array}$

180830065284900

180838065282500

$2-3$

3 Martineau dug well

180834065281600

$2-4$
3 Corcino-Meléndez dug well

$\begin{array}{lll}3-1 & 4 & \text { Iglesia Metodista } \\ 3-2 & 4 & \text { Castaño dug well }\end{array}$

3-3

$3-4$

$\begin{array}{ll}4 & \text { Metodista } 1 \\ 4 & \text { G. Colón }\end{array}$

3-5
4 Agrícola Corp. 2 dug well

PRASA 10 dug well

4 F. Peterson dug well

$5-1$

$$
6 \text { Camp García dug well }
$$

$\begin{array}{lll}9-1 & 10 & \text { U.S. Navy } 17\end{array}$

10-1 Pilón 1 dug well
180816065214800

180727065312600

180833065275100

180828065265000

180844065263100

180859065261200

180918065261400

180816065260300

180810065255800

180733065265800

Well used in the past for potable water. Water sample for specific conductance and chloride concentration bailed from a depth of 5 feet below the water table.

Water sample for specific conductance and chloride concentration bailed from a depth of 1 foot below the water table.

Water sample for specific conductance and chloride concentration bailed from a depth of 10 feet below the water table.

$180631065295800 \quad$ Well casing (6 inch PVC) placed inside a 72-inch diameter. Water sample for specific conductance and chloride concentration bailed from a depth of 4 feet below the water table. Submersible pump installed. 
Appendix 1. Well names, site-identification number, and remarks for wells on Isla de Vieques, Puerto Rico—Continued

\begin{tabular}{|c|c|c|c|c|}
\hline $\begin{array}{l}\text { Well } \\
\text { No. }\end{array}$ & $\begin{array}{c}\text { Figure } \\
\text { No. }\end{array}$ & Well name & Site identification No. & Remarks \\
\hline $10-2$ & 11 & Sucn Díaz 1 dug well & 180659065293800 & $\begin{array}{l}\text { Water sample for specific conductance and chloride } \\
\text { concentration bailed from a depth of } 6 \text { feet below the } \\
\text { water table. }\end{array}$ \\
\hline $10-3$ & 11 & O. González & 180636065291900 & $\begin{array}{l}\text { Measured yield of } 8 \text { gallons per minute. Surface pump } \\
\text { installed. Water sample for specific conductance and } \\
\text { chloride concentration taken from the discharge line. }\end{array}$ \\
\hline $10-4$ & 11 & Vieques 12 & 180638065284000 & $\begin{array}{l}\text { Water sample for specific conductance and chloride } \\
\text { concentration bailed from a depth of } 6 \text { feet below the } \\
\text { water table. }\end{array}$ \\
\hline $10-5$ & 11 & U.S. Navy 15 dug well & 180709065282700 & - \\
\hline $10-6$ & 11 & Vieques 8 & 180622065273100 & $\begin{array}{l}\text { U.S. Geological Survey observation well. Water } \\
\text { sample for specific conductance and chloride } \\
\text { concentration bailed from a depth of } 20 \text { feet below the } \\
\text { water table. }\end{array}$ \\
\hline $10-7$ & 11 & PRASA 1 & 180641065280700 & -- \\
\hline $10-8$ & 11 & USGS La Mina & 180643065280700 & $\begin{array}{l}\text { U.S. Geological Survey observation well. Water } \\
\text { sample for specific conductance and chloride } \\
\text { concentration bailed from a depth of } 5 \text { feet below the } \\
\text { water table. }\end{array}$ \\
\hline $10-9$ & 11 & PRASA 2 & 180648065280600 & - \\
\hline $10-10$ & 11 & PRASA 3 & 180641065280100 & - \\
\hline $10-11$ & 11 & La Mina 1 & 180626065280200 & U.S. Geological Survey observation well. \\
\hline $10-12$ & 11 & La Mina 2 & 180625065280100 & $\begin{array}{l}\text { U.S. Geological Survey observation well. Water } \\
\text { sample for specific conductance and chloride } \\
\text { concentration bailed from a depth of } 20 \text { feet below the } \\
\text { water table. }\end{array}$ \\
\hline $10-13$ & 11 & Fesa 2 dug well & 180601065281100 & $\begin{array}{l}\text { Well used by the sugarcane industry in the past. Water } \\
\text { sample for specific conductance and chloride } \\
\text { concentration bailed from a depth of } 6 \text { feet below the } \\
\text { water table. }\end{array}$ \\
\hline $10-14$ & 11 & O'Conner 12 & 180605065280300 & $\begin{array}{l}\text { Water sample for specific conductance and chloride } \\
\text { concentration bailed from a depth of } 10 \text { feet below the } \\
\text { water table. }\end{array}$ \\
\hline $10-15 a$ & 11 & PRASA A-5 & 180559065280700 & Well clogged with soil and debris. \\
\hline $10-15 b$ & 11 & PRASA A-5A & 180559065280701 & Well is 9 feet from PRASA A- 5 well. \\
\hline $10-16$ & 11 & PRASA A-4 & 180559065280600 & Well clogged with soil and debris. \\
\hline $10-17 \mathrm{a}$ & 11 & PRASA A-2 & 180559065280500 & $\begin{array}{l}\text { Submersible pump installed. Drawdown was } 2.4 \text { feet } \\
\text { after } 18 \text { hours pumping } 25 \text { gallons per minute (March } \\
26-27,1992 \text { ). }\end{array}$ \\
\hline
\end{tabular}


Appendix 1. Well names, site-identification number, and remarks for wells on Isla de Vieques, Puerto Rico-Continued

\begin{tabular}{lcccc}
\hline Well & Figure & Well name & Site identification No. & Remarks \\
No. & No. & N
\end{tabular}

10-17b $11 \quad$ PRASA A-3

10-18 $11 \quad$ PRASA A-1

180559065280400

$10-19$

11 USGS piezometer 1

180600065280400

10-20 $11 \quad$ Vieques 5A

180605065275900

10-21 $11 \quad$ PRASA 4

180612065275500

$10-22 \quad 11 \quad$ Vieques 5

10-23 $11 \quad$ PRASA D-3

10-24a $11 \quad$ PRIDCO 9A

10-24b $11 \quad$ PRIDCO 9

10-25 $11 \quad$ PRASA 151

180559065280501

180607065275600

180608065275400

180558065275000

180558065275001
Well is 50 feet from PRASA A-2 well. Submersible pump installed. Drawdown was 4.3 feet after 8 hours pumping 25 gallons per minute (March 19, 1992).

Wells PRASA A-1-3 provided water to the public in the aftermath of Hurricane Hugo ${ }^{1}$. Water sample for specific conductance and chloride concentration bailed from a depth of 20 feet below the water table.

Drawdown was 1.5 feet after 8 hours pumping 25 gallons per minute (March 11, 1992).

Water sample for specific conductance and chloride concentration bailed from a depth of 10 feet below the water table.

U.S. Geological Survey observation well. Water sample for specific conductance and chloride concentration bailed from a depth of 30 feet below the water table.

Water sample for specific conductance and chloride concentration bailed from a depth of 10 feet below the water table.

U.S. Geological Survey observation well. Water sample for specific conductance and chloride concentration bailed from a depth of 20 feet below the water table.

Submersible pump installed. Drawdown was 1.7 feet after 21 hours pumping 25 gallons per minute (March 26-27, 1992).

U.S. Geological Survey observation well. Well seems to be clogged or collapsed. Water sample for specific conductance and chloride concentration bailed from a depth of 1 foot below the water table. Analog digital recorder installed to monitor daily and seasonal fluctuations of the water level.

U.S. Geological Survey observation well. Water sample for specific conductance and chloride concentration bailed from a depth of 70 feet below the water table. Analog digital recorder installed to monitor daily and seasonal fluctuations of the water level.

Water sample for specific conductance and chloride concentration bailed from a depth of 1 foot below the water table. 
Appendix 1. Well names, site-identification number, and remarks for wells on Isla de Vieques, Puerto Rico-Continued

\begin{tabular}{|c|c|c|c|c|}
\hline $\begin{array}{l}\text { Well } \\
\text { No. }\end{array}$ & $\begin{array}{c}\text { Figure } \\
\text { No. }\end{array}$ & Well name & Site identification No. & Remarks \\
\hline $10-26$ & 11 & PRASA D-2 & 180613065274600 & $\begin{array}{l}\text { Submersible pump installed. Drawdown was } 1.6 \text { feet } \\
\text { after } 8 \text { hours pumping } 25 \text { gallons per minute (April 1, } \\
\text { 1992). }\end{array}$ \\
\hline $10-27$ & 11 & PRASA 5 & 180616065274700 & Submersible pump installed. \\
\hline $10-28$ & 11 & Vieques 18 & 180614065274700 & $\begin{array}{l}\text { U.S. Geological Survey observation well. Water } \\
\text { sample for specific conductance and chloride } \\
\text { concentration bailed from a depth of } 25 \text { feet below the } \\
\text { water table. }\end{array}$ \\
\hline $10-29$ & 11 & PRASA 6 & 180618065274400 & $\begin{array}{l}\text { Water sample for specific conductance and chloride } \\
\text { concentration bailed from a depth of } 20 \text { feet below the } \\
\text { water table. }\end{array}$ \\
\hline $10-30$ & 11 & PRASA D-1 & 180616065274000 & $\begin{array}{l}\text { Submersible pump installed. Drawdown was } 2.4 \text { feet } \\
\text { after } 8 \text { hours pumping } 25 \text { gallons per minute (April } 1 \text {, } \\
\text { 1992). }\end{array}$ \\
\hline $10-31$ & 11 & PRASA 7 & 180616065274600 & $\begin{array}{l}\text { Water sample for specific conductance and chloride } \\
\text { concentration bailed from a depth of } 2 \text { feet below the } \\
\text { water table. }\end{array}$ \\
\hline $10-32$ & $\cdot 11$ & Vieques 22 & 180615065271900 & $\begin{array}{l}\text { U.S. Geological Survey observation well. Water } \\
\text { sample for specific conductance and chloride } \\
\text { concentration bailed from a depth of } 30 \text { feet below the } \\
\text { water table. }\end{array}$ \\
\hline $10-33$ & 11 & PRASA B-4 & 180621065274900 & $\begin{array}{l}\text { Submersible pump installed. Drawdown was } 0.8 \text { foot } \\
\text { after } 4 \text { hours pumping } 25 \text { gallons per minute (April } 2 \text {, } \\
\text { 1992). }\end{array}$ \\
\hline $10-34$ & 11 & PRASA B-3 & 180621065274901 & $\begin{array}{l}\text { Submersible pump installed. Drawdown was } 5.9 \text { feet } \\
\text { after } 24 \text { hours pumping } 62 \text { gallons per minute (March } \\
31 \text { to April 1, 1992). }\end{array}$ \\
\hline $10-35$ & 11 & PRASA B-2 & 180621065274902 & $\begin{array}{l}\text { Submersible pump installed. Drawdown was } 6.8 \text { feet } \\
\text { after } 8 \text { hours pumping } 25 \text { gallons per minute (April } 3 \text {, } \\
\text { 1992). }\end{array}$ \\
\hline $10-36$ & 11 & PRASA B-1 & 180621065274903 & $\begin{array}{l}\text { Submersible pump installed. Drawdown was } 3.9 \text { feet } \\
\text { after } 8 \text { hours pumping } 25 \text { gallons per minute (April } 2 \text {, } \\
\text { 1992). }\end{array}$ \\
\hline $11-1$ & 12 & PRASA C-3 & 180625065272500 & $\begin{array}{l}\text { Broken water line next to well. It is likely that water } \\
\text { from the broken water-supply line has mixed with the } \\
\text { well water. Water sample for specific conductance and } \\
\text { chloride concentration bailed from a depth of } 20 \text { feet } \\
\text { below the water table. Reported depth of well from } \\
\text { PRASA records of } 82 \text { feet. }\end{array}$ \\
\hline
\end{tabular}


Appendix 1. Well names, site-identification number, and remarks for wells on Isla de Vieques, Puerto Rico-Continued

\begin{tabular}{|c|c|c|c|c|}
\hline $\begin{array}{l}\text { Well } \\
\text { No. }\end{array}$ & $\begin{array}{l}\text { Figure } \\
\text { No. }\end{array}$ & Well name & Site identification No. & Remarks \\
\hline $11-2$ & 12 & PRASA C-2 & 180626065272500 & $\begin{array}{l}\text { Water sample for specific conductance and chloride } \\
\text { concentration bailed from a depth of } 35 \text { feet below the } \\
\text { water table. }\end{array}$ \\
\hline $11-3$ & 12 & PRASA C-1 & 180628065272300 & $\begin{array}{l}\text { Water sample for specific conductance and chloride } \\
\text { concentration bailed from a depth of } 28 \text { feet below the } \\
\text { water table. }\end{array}$ \\
\hline $11-4$ & 12 & Vieques 14 & 180631065272000 & $\begin{array}{l}\text { U.S. Geological Survey observation well. Unable to } \\
\text { sample or measure depth of well due to obstruction } \\
\text { inside casing. }\end{array}$ \\
\hline $11-5$ & 12 & Vieques 16 & 180630065271300 & $\begin{array}{l}\text { U.S. Geological Survey observation well. Water } \\
\text { sample for specific conductance and chloride } \\
\text { concentration bailed from a depth of } 5 \text { feet below the } \\
\text { water table. }\end{array}$ \\
\hline $11-6$ & 12 & Vieques 17 & 180636065271300 & $\begin{array}{l}\text { Well constructed by the U.S. Geological Survey. } \\
\text { Submersible pump installed. }\end{array}$ \\
\hline $11-7$ & 12 & Vieques 15 & 180650065271400 & $\begin{array}{l}\text { U.S. Geological Survey observation well. Water } \\
\text { sample for specific conductance and chloride } \\
\text { concentration bailed from a depth of } 10 \text { feet below the } \\
\text { water table. }\end{array}$ \\
\hline $11-8$ & 12 & Vieques $8 \mathrm{~A}$ & 180654065270800 & $\begin{array}{l}\text { Well constructed by the U.S. Geological Survey. } \\
\text { Reported yield of } 20 \text { gallons per minute. Submersible } \\
\text { pump installed at a depth of } 55 \text { feet below land } \\
\text { surface. Water sample for specific conductance and } \\
\text { chloride concentration taken from the discharge line. }\end{array}$ \\
\hline $11-9$ & 12 & O’Conner 4 & 180644065265500 & $\begin{array}{l}\text { Water sample for specific conductance and chloride } \\
\text { concentration bailed from a depth of } 4 \text { feet below the } \\
\text { water table. }\end{array}$ \\
\hline $11-10$ & 12 & Vieques 1 & 180657065264600 & $\begin{array}{l}\text { U.S. Geological Survey observation well. Water } \\
\text { sample for specific conductance and chloride } \\
\text { concentration bailed from a depth of } 35 \text { feet below the } \\
\text { water table. }\end{array}$ \\
\hline $11-11$ & 12 & O'Conner 5 & 180647065264800 & -- \\
\hline $11-12$ & 12 & Land Authority dug well & 180723065263300 & $\begin{array}{l}\text { Well was used in the past for potable water by the } \\
\text { Lujan community. Water sample for specific } \\
\text { conductance and chloride concentration bailed from a } \\
\text { depth of } 3 \text { feet below the water table. }\end{array}$ \\
\hline $11-13$ & 12 & Vieques 19 & 180719065261900 & $\begin{array}{l}\text { U.S. Geological Survey observation well. Water } \\
\text { sample for specific conductance and chloride } \\
\text { concentration bailed from a depth of } 2 \text { feet below the } \\
\text { water table. Well may be blocked at } 46 \text { feet. }\end{array}$ \\
\hline
\end{tabular}


Appendix 1. Well names, site-identification number, and remarks for wells on Isla de Vieques, Puerto Rico-Continued

\begin{tabular}{|c|c|c|c|c|}
\hline $\begin{array}{l}\text { Well } \\
\text { No. }\end{array}$ & $\begin{array}{c}\text { Figure } \\
\text { No. }\end{array}$ & Well name & Site identification No. & Remarks \\
\hline $11-14$ & 12 & U.S. Marines 6 & 180717065260900 & $\begin{array}{l}\text { This well, combined with U.S. Navy } 14 \text { well (well } 11 \text { - } \\
\text { 17), pumps about } 84,000 \text { gallons per day twice a week } \\
\text { for use by the Camp García personnel. Submersible } \\
\text { pump installed at a depth of } 65 \text { feet below land } \\
\text { surface. }\end{array}$ \\
\hline $11-15$ & 12 & U.S. Navy 13 & 180715065260800 & $\begin{array}{l}\text { Unable to measure well depth or sample due to bees' } \\
\text { nest inside well casing. }\end{array}$ \\
\hline $11-16$ & 12 & U.S. Navy Camp García & 180716065261100 & $\begin{array}{l}\text { Water sample for specific conductance and Camp } \\
\text { García chloride concentration bailed from a depth of } 6 \\
\text { feet below the water table. }\end{array}$ \\
\hline $11-17$ & 12 & U.S. Navy 14 & 180714065260800 & $\begin{array}{l}\text { This well combined with U.S. Marines } 6 \text { well (well 11- } \\
\text { 14) pumps about } 84,000 \text { gallons per day twice a week } \\
\text { for use by the Camp García personnel. Submersible } \\
\text { pump installed at a depth of } 65 \text { feet below land } \\
\text { surface. }\end{array}$ \\
\hline $11-18$ & 12 & USGS-piezometer 2 & 180712065260800 & -- \\
\hline $11-19$ & 12 & Vieques 2 & 180702065262000 & $\begin{array}{l}\text { U.S. Geological Survey observation well. Water } \\
\text { sample for specific conductance and chloride } \\
\text { concentration bailed at a depth of } 30 \text { feet below the } \\
\text { water table. }\end{array}$ \\
\hline $11-20$ & 12 & Vieques 4 & 180652065261700 & $\begin{array}{l}\text { U.S. Geological Survey observation well. Water } \\
\text { sample for specific conductance and chloride } \\
\text { concentration bailed at a depth of } 1 \text { foot below the } \\
\text { water table. Well may be blocked at } 29 \text { feet. }\end{array}$ \\
\hline $11-21$ & 12 & U.S. Marines 4 & 180721065251200 & $\begin{array}{l}\text { Submersible pump installed. Water sample for specific } \\
\text { conductance and chloride concentration bailed from a } \\
\text { depth of } 20 \text { feet below the water table. }\end{array}$ \\
\hline
\end{tabular}

\footnotetext{
${ }^{1}$ Hurricane Hugo struck Isla de Vieques, Puerto Rico, during the early morning hours of September 18, 1989.
} 